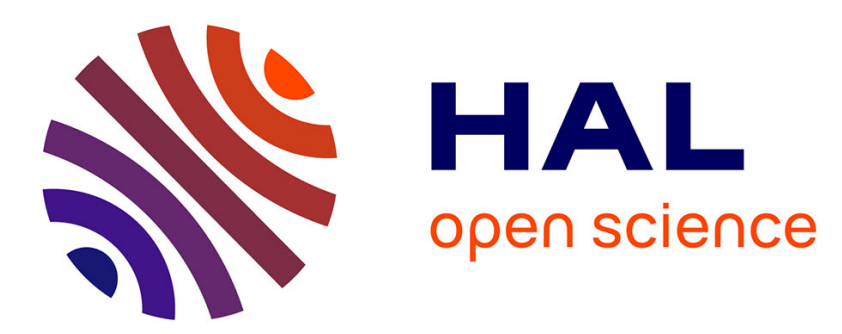

\title{
Ab initio potential energy curve for the helium atom pair and thermophysical properties of the dilute helium gas. II. Thermophysical standard values for low-density helium
}

Eckhard Vogel, Eckard Bich, Robert Hellmann

\section{To cite this version:}

Eckhard Vogel, Eckard Bich, Robert Hellmann. Ab initio potential energy curve for the helium atom pair and thermophysical properties of the dilute helium gas. II. Thermophysical standard values for low-density helium. Molecular Physics, 2007, 105 (23-24), pp.3035-3049. 10.1080/00268970701744584. hal-00513156

\author{
HAL Id: hal-00513156 \\ https://hal.science/hal-00513156
}

Submitted on 1 Sep 2010

HAL is a multi-disciplinary open access archive for the deposit and dissemination of scientific research documents, whether they are published or not. The documents may come from teaching and research institutions in France or abroad, or from public or private research centers.
L'archive ouverte pluridisciplinaire HAL, est destinée au dépôt et à la diffusion de documents scientifiques de niveau recherche, publiés ou non, émanant des établissements d'enseignement et de recherche français ou étrangers, des laboratoires publics ou privés. 


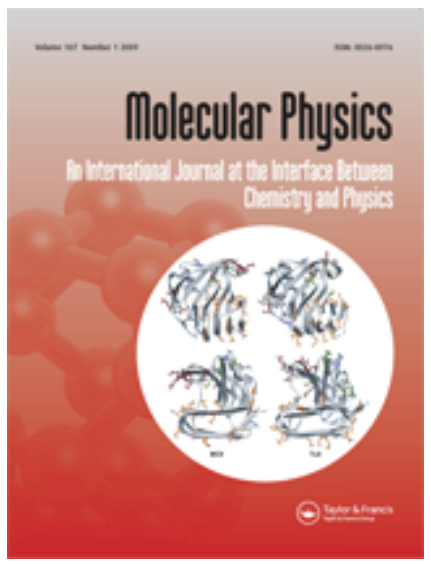

\section{Ab initio potential energy curve for the helium atom pair and thermophysical properties of the dilute helium gas. II. Thermophysical standard values for low-density helium}

\begin{tabular}{|r|l|}
\hline Journal: & Molecular Physics \\
\hline Manuscript ID: & TMPH-2007-0246.R1 \\
\hline Manuscript Type: & Full Paper \\
\hline Duthor: & 27-Sep-2007 \\
\hline Complete List of Authors: & $\begin{array}{l}\text { Vogel, Eckhard; Universität Rostock, Institut für Chemie; } \\
\text { Universität Rostock, Institut für Chemie } \\
\text { Bich, Eckard; Universität Rostock, Institut für Chemie } \\
\text { Hellmann, Robert; Universität Rostock, Institut für Chemie }\end{array}$ \\
\hline Keywords: & $\begin{array}{l}\text { Helium pair potential, helium gas property standards, second and } \\
\text { third pressure virial coefficients, viscosity, thermal conductivity }\end{array}$ \\
\hline \multicolumn{2}{|c|}{} \\
\hline Note: The following files were submitted by the author for peer review, but cannot be converted \\
to PDF. You must view these files (e.g. movies) online.
\end{tabular}

\section{S) ScholaroNE \\ Manuscript Central}




\title{
$A b$ initio potential energy curve for the helium atom pair and thermophysical properties of the dilute helium gas. \\ II. Thermophysical standard values for low-density helium
}

\author{
ECKARD BICH, ROBERT HELLMANN, and ECKHARD VOGEL* \\ Institut für Chemie, Universität Rostock, Albert-Einstein-Straße 3a, D-18059 Rostock, \\ Germany \\ (Received 00 Month 200x; in final form 00 Month 200x)
}

\begin{abstract}
A helium-helium interatomic potential energy curve determined from quantum-mechanical $a b$ initio calculations and described with an analytical representation considering relativistic retardation effects (R. Hellmann, E. Bich, and E. Vogel, Mol. Phys. (submitted)) was used in the framework of the quantum-statistical mechanics and of the corresponding kinetic theory to calculate the most important thermophysical properties of helium governed by two-body and three-body interactions. The second pressure virial coefficient as well as the viscosity and thermal conductivity coefficients, the last two in the so-called limit of zero density, were calculated for ${ }^{3} \mathrm{He}$ and ${ }^{4} \mathrm{He}$ from $1 \mathrm{~K}$ to $10,000 \mathrm{~K}$ and the third pressure virial coefficient for ${ }^{4} \mathrm{He}$ from $20 \mathrm{~K}$ to $10,000 \mathrm{~K}$. The transport property values can be applied as standard values for the complete temperature range of the calculations characterized by an uncertainty of $\pm 0.02 \%$ for temperatures above $15 \mathrm{~K}$. This uncertainty is superior to the best experimental measurements at ambient temperature.
\end{abstract}

Keywords: Helium pair potential; helium gas property standards; second and third pressure virial coefficients; viscosity; thermal conductivity

\section{Introduction}

Hurly and Moldover [1] as well as Hurly and Mehl [2] stated that standard values of the thermophysical properties of helium at low densities which can be used for different applications in metrology and for the calibration of measuring instruments are derived best from the helium-helium interatomic potential energy curve. For that purpose the interatomic potential has to be determined from quantummechanical ab initio calculations and should be described by a suitable analytical representation. Then the thermophysical properties at low density should follow from calculations using the kinetic theory of gases together with standard formulae from quantum-statistical mechanics. Furthermore, Hurly, Moldover, and Mehl

*Corresponding author. Email: eckhard.vogel@uni-rostock.de 


\section{Molecular Physics}

established that the uncertainties of the calculated thermophysical property values, such as second pressure and dielectric virial coefficients, viscosity and thermal conductivity coefficients, speed of sound, and further properties, are smaller than the corresponding uncertainties of the experimental data, even for temperatures at which high-precision measurements can comparably easily be performed.

In our paper I [3] a new helium-helium interatomic potential energy curve was determined for a comparably large number of interatomic separations from quantummechanical $a b$ initio calculations using very large atom-centred basis sets, including a newly developed d-aug-cc-pV8Z basis set supplemented with bond functions, and $a b$ initio methods up to Full CI. The diagonal Born-Oppenheimer corrections as well as corrections for relativistc effects were also enclosed. An improved analytical representation of the interatomic potential energy was fitted to the new $a b$ initio calculated values and to some from the literature. Hurly and Mehl constructed their potential from literature values only. Some of these values are nearly as accurate as the new values from paper I, but they are only available for very few interatomic separations. Hence Hurly and Mehl had to use significantly less accurate values for most of the helium-helium distances. It should also be stressed that their analytical representation of the potential function is less flexible than the one used in our paper I. As a result the analytical potential of Hurly and Mehl is characterized by comparably large fitting errors in the regions of the potential to which the thermophysical properties are most sensitive. For the potential of paper I the fitting errors are nearly negligible in these regions (see table 5 of paper I).

In this contribution the new helium-helium interatomic potential model has been used in the framework of the quantum-statistical mechanics and of the corresponding kinetic theory to calculate the most important thermophysical properties of helium governed by two-body and three-body interactions. In a second series of papers the investigation shall be extended to neon in order to generate standard values of the thermophysical properties for a second substance to be used for the calibration of measuring instruments.

\section{Analytical helium-helium potential function}

The ab initio calculated interatomic potential energy values $V(R)$ including some relativistic corrections and the diagonal Born-Oppenheimer corrections, but without retardation, which were chosen for the fit of the analytical potential function, and the fitted unretarded potential values have been listed in Table 5 of paper I [3]. A modification of the potential function given by Tang and Toennies [4] was used 
as potential model:

$$
\begin{aligned}
V(R) & =A \exp \left(a_{1} R+a_{2} R^{2}+a_{-1} R^{-1}+a_{-2} R^{-2}+d_{1} \sin \left(d_{2} R+d_{3}\right)\right) \\
& -\sum_{n=3}^{8} f_{2 n}(R) \frac{C_{2 n}}{R^{2 n}}\left[1-\exp (-b R) \sum_{k=0}^{2 n} \frac{(b R)^{k}}{k !}\right]
\end{aligned}
$$

Whereas the details of the fit (with $f_{2 n}(R)=1$ for all $n$ ) were communicated in paper I, the potential parameters are repeatedly given for convenience in Table 1 .

The retardation effects, which change for asymptotic separations the $C_{6} / R^{6}$ behaviour of the potential into $C_{7} / R^{7}$ as demonstrated by Casimir and Polder [5] and which are also of importance for the only vibrational state of ${ }^{4} \mathrm{He}[6-8]$, have to be included into the representation of the helium-helium interaction potential used for the calculation of the thermophysical properties under discussion. The functions $f_{2 n}(R)$ take into consideration for all separations the relativistic retardation of the dipole-dipole term as well as of the next higher dispersion terms with $n=3-5[9-11]$. The approximation $f_{2 n}(R)=1$ was used for the further $n>5$. The $f_{2 n}(R)$ values given in [11] were interpolated using Lagrange's polynomial for 5 points and implemented for the potential after the fit. The retardation correction (i.e. the difference between the retarded and the unretarded potentials) is also listed in Table 5 of paper I. The potential parameters $\varepsilon / k_{\mathrm{B}}, R_{\varepsilon}$, and $\sigma$ for the retarded potential are given in Table 1 , too.

\section{Quantum-mechanical calculation of thermophysical properties}

Very accurate values for the thermophysical properties of helium can only be gained by a fully quantum-mechanical treatment of the elastic scattering considering the interatomic potential $V(R)$. The eigenfunction of a particle with the reduced mass $\mu=\left(m_{1} m_{2}\right) /\left(m_{1}+m_{2}\right)$ related to the centre of mass can be expressed as the infinite sum over partial waves, each of them corresponds to a particular state of the angular momentum of the system. The Schrödinger equation for the radial factor $\psi_{l}(R)$ of the $l$ th partial wave with the angular momentum quantum number $l$ and the wave number $k=(2 \mu E)^{1 / 2} / \hbar$ is given as

$$
\left(\frac{\mathrm{d}^{2}}{\mathrm{~d} R^{2}}+k^{2}-\frac{2 \mu}{\hbar^{2}} V(R)-\frac{l(l+1)}{R^{2}}\right) \psi_{l}(R)=0 .
$$

Here $E$ is the energy of the incoming wave, $\hbar$ is Planck's constant $h$ divided by $2 \pi$.

It is to be stressed that the reduced mass results from the atomic masses in the framework of the Born-Oppenheimer approximation following the discussion by Handy and Lee [12] as well as Kutzelnigg [13]. 


\section{Molecular Physics}

\subsection{Evaluation of the phase shifts}

To calculate the thermophysical properties of helium the relative phase shifts $\delta_{l}$ are needed. They correspond to the difference in the relative phase of the radial part of the outgoing wave functions $\psi_{l}(R)$ and $\psi_{l}^{(0)}(R)$. Here $\psi_{l}(R)$ is perturbed by the influence of the interatomic potential $V(R)$, whereas $\psi_{l}^{(0)}(R)$ is unperturbed, i.e. $V(R)=0$. The phase shifts $\delta_{l}$ have to be evaluated as asymptotic limiting values of the relative phases of the perturbed and unperturbed waves. For that purpose nodes of the outgoing waves located at $R_{n}$ of the $n$th zero far from the scattering centre have to be used. McConville and Hurly [14] discussed problems in the evaluation of the phase shifts in connection with two codes available in the literature $[15,16]$ and recommended to determine the phase shifts using the relation

$$
\delta_{l}^{\prime}(k, n)=\arctan \frac{\mathrm{j}_{l}\left(k, R_{n}\right)}{\mathrm{n}_{l}\left(k, R_{n}\right)}
$$

Here $\mathrm{j}_{l}\left(k, R_{n}\right)$ and $\mathrm{n}_{l}\left(k, R_{n}\right)$ are Bessel and Neumann functions for the angular momentum quantum number $l$ and the wave number $k$. In the asymptotic limit the phase shift becomes independent of the node number. The numerical integration was performed from node to node and was stopped when the change of the phase shifts $\left|\Delta \delta_{l}^{\prime}(k, n)\right|$ between two successive nodes became smaller than $10^{-9}$. Because of the restricted range of the arctan function the phase shifts $\delta_{l}^{\prime}(k)$ resulting from Eq. (3) have to be corrected by an integer multiple of $\pi$ in order to get the true values:

$$
\delta_{l}(k)=\delta_{l}^{\prime}(k, n)+n_{\pi} \pi
$$

The value $n_{\pi}$ follows from

$$
n_{\pi}=n-\left\lfloor\frac{\theta_{l}+\delta_{l}^{\prime}}{\pi}+0.5\right\rfloor
$$

with

$$
\begin{aligned}
\theta_{l} & \approx x-\left(\frac{1}{2} l+\frac{1}{4}\right) \pi+\frac{\lambda-1}{2(4 x)}+\frac{(\lambda-1)(\lambda-25)}{6(4 x)^{3}}+\frac{(\lambda-1)\left(\lambda^{2}-114 \lambda+1073\right)}{5(4 x)^{5}} \\
& +\frac{(\lambda-1)\left(5 \lambda^{3}-1535 \lambda^{2}+54703 \lambda-375733\right)}{14(4 x)^{7}}+\cdots
\end{aligned}
$$

and

$$
\lambda=4 l^{2} \quad x=k R_{n} .
$$

$\theta_{l}$ represents the phase of the partial wave $\psi_{l}^{(0)}(R)$ in the asymptotic limit (Eq. 9.2.29 in [17]) of the ideal system. 
The fully quantum-mechanical calculation of the phase shifts at a multiplicity of wave numbers $k$ for a large number of $l$ values is very expensive with respect to the computing time. Hence it is reasonable to minimize this time by using suitable approximations, such as the JWKB method. In this semi-classical approximation the phase shifts result from

$$
\begin{aligned}
\delta_{l}(k) & =\frac{(2 \mu)^{\frac{1}{2}}}{\hbar}\left\{\int_{R_{1}}^{R_{2}}\left(\frac{\hbar^{2} k^{2}}{2 \mu}-\frac{l(l+1) \hbar^{2}}{2 \mu R^{2}}-V(R)\right)^{\frac{1}{2}} \mathrm{~d} R\right. \\
& +\int_{R_{3}}^{\infty}\left(\frac{\hbar^{2} k^{2}}{2 \mu}-\frac{l(l+1) \hbar^{2}}{2 \mu R^{2}}-V(R)\right)^{\frac{1}{2}} \mathrm{~d} R \\
& \left.-\int_{R_{0}}^{\infty}\left(\frac{\hbar^{2} k^{2}}{2 \mu}-\frac{l(l+1) \hbar^{2}}{2 \mu R^{2}}\right)^{\frac{1}{2}} \mathrm{~d} R\right\} .
\end{aligned}
$$

Here $R_{1}, R_{2}$, and $R_{3}$ correspond to the three roots of the separation after equating the energy with the effective potential characterized by a centrifugal barrier at small and medium $l$ values. In the case that the centrifugal barrier disappears at high $l$ values as well as in the case that the energy is higher than the centrifugal barrier, only one root occurs and the first integral in Eq. (7) can be neglected. This corresponds to the usual procedure in the classical treatment of the scattering to use only the outer root. $R_{0}$ is the smallest separation in the case that there is no influence of the interatomic potential $V(R)$.

The calculation of the phase shifts $\delta_{l}(k)$ was performed for 585 values of the energy $E$ in the range from zero to $250,000 \mathrm{~K}$ and for a number of $l$ values increasing with rising energy. The phase shifts were determined fully quantum-mechanically using Eqs. (3) to (6) as long as their values did not become too small. Parallel to it phase shifts according to the JWKB approximation using Eq. (7) were calculated, and their results were compared with those of the fully quantum-mechanical evaluation. In the case that the values of both procedures came into close agreement for certain values of the angular momentum quantum number $l$, the fully quantummechanical evaluation (QM) was replaced by the semi-classical JWKB procedure at the higher $l$ values. The number of phase shifts which were evaluated according to both procedures and used in the further calculations are listed for some reduced energies $E^{*}=E / \varepsilon$ in Table 2 . The large number of phase shifts has been chosen to avoid uncertainties in the results of the calculated thermophysical properties. This applies particularly to the second virial coefficient discussed next.

\subsection{Calculation of the second pressure virial coefficient}

The second virial coefficient is given following Boyd et al. [18] in two contributions: $B_{\text {direct }}$ and $B_{\text {exch. }}$ This separation is reasonable, because the effects due to symmetry are explicitly displayed and the role of spin is demonstrated in a simple 


\section{Molecular Physics}

manner. $B_{\text {direct }}$ and $B_{\text {exch }}$ can be represented by means of summations over only the even $l$ values and only the odd $l$ values:

$$
\begin{aligned}
B_{\text {direct }} & =B_{\text {even }}+B_{\text {odd }}, \\
B_{\text {exch }} & =\left(\frac{1}{2 s+1}\right)\left(B_{\text {even }}-B_{\text {odd }}-\frac{N_{\mathrm{A}} \Lambda^{3}}{16}\right)
\end{aligned}
$$

following from the relationship:

$$
\begin{aligned}
B_{l}(T) & =-\frac{N_{\mathrm{A}} \Lambda^{3}}{2}\left[\sum_{n=0}^{n_{\max } l_{\max }(n)} \sum_{l}(2 l+1)\left(\mathrm{e}^{-\beta E_{n l}^{-}}-1\right)\right. \\
& \left.+\int_{0}^{\infty} \sum_{l}^{\infty}(2 l+1) \frac{\delta_{l}(E)}{\pi} \mathrm{e}^{-\beta E} \mathrm{~d}(\beta E)\right] .
\end{aligned}
$$

Here $\Lambda$ is the thermal wave length:

$$
\Lambda=\left(\frac{h^{2} \beta}{2 \pi \mu}\right)^{\frac{1}{2}}, \quad \beta=\frac{1}{k_{\mathrm{B}} T} .
$$

The spin quantum number is $s=1 / 2$ for ${ }^{3} \mathrm{He}$ and $s=0$ for ${ }^{4} \mathrm{He}$, hence ${ }^{3} \mathrm{He}$ is a Fermion and ${ }^{4} \mathrm{He}$ is a Boson. The third term in Eq. (9) represents the ideal-gas term which is only important at low temperatures. $B_{\text {exch }}$, considering spin and quantum statistics, goes rapidly to zero with increasing temperature. The first term of Eq. (10) corresponds to the contribution of the bound states, where $E_{n l}^{-}$ is the negative eigenvalue of the $n$th state with the angular-momentum quantum number $l$ which is obtained from the solution of the Schrödinger equation for the radial factor of a partial wave. It is to note that there exists no bound state for the ${ }^{3} \mathrm{He}-{ }^{3} \mathrm{He}$ pair, whereas only one bound state occurs for ${ }^{4} \mathrm{He}-{ }^{4} \mathrm{He}$ about $1 \mathrm{mK}$ below the dissociation limit [6-8]. The bound state contribution is only of importance at very low temperatures in the case of ${ }^{4} \mathrm{He}$. The second term of Eq. (10) is the most important contribution at medium and higher temperatures and is related to the scattering resulting from binary collisions and to the phase shifts $\delta_{l}$.

The term $B_{\text {direct }}$ which corresponds to the complete summation over all $l$ values in Eq. (10) corresponds to the Boltzmann statistics:

$$
B_{\mathrm{B}}=B_{\text {direct }}
$$

whereas for particles with spin $s$ according to the Bose-Einstein (BE) or to the Fermi-Dirac (FD) statistics holds:

$$
\begin{aligned}
& B_{\mathrm{BE}}=B_{\text {direct }}+B_{\text {exch }}, \\
& B_{\mathrm{FD}}=B_{\text {direct }}-B_{\text {exch }} .
\end{aligned}
$$




\section{Molecular Physics}

II. Thermophysical standard values for low-density helium

The sum over $l$ and the integral in Eq. (10) have limits from 0 to $\infty$ and could lead to serious errors in the computation when truncated inadequately. Hence it was tested that the energies for which the calculations were performed and particularly the number of the phase shifts were chosen largely enough (see Table 2).

\subsection{Calculation of the third pressure virial coefficient}

To obtain the third virial coefficient the three-body interatomic interaction potential $V_{3}\left(R_{12}, R_{13}, R_{23}\right)$ is needed. If it is assumed that apart from the pairwise additivity of the two-body interatomic potentials an extra genuine term $C_{\text {non-add }}$ for the non-additivity $\Delta V_{3}\left(R_{12}, R_{13}, R_{23}\right)$ occurs and quantum effects as a first-order correction $C_{\mathrm{qm}, 1}$ are taken into account, the third virial coefficient is calculated as a sum of three contributions $[19,20]$ :

$$
\begin{aligned}
C_{\text {add }} & =-6 b_{0}^{2} \int_{0}^{\infty}\left(\mathrm{e}^{-\beta V\left(R_{12}\right)}-1\right) R_{12}^{2} \int_{0}^{\infty}\left(\mathrm{e}^{-\beta V\left(R_{13}\right)}-1\right) R_{13}^{2} \\
& \int_{-1}^{1}\left(\mathrm{e}^{-\beta V\left(R_{23}\right)}-1\right) \mathrm{d} X \mathrm{~d} R_{13} \mathrm{~d} R_{12}, \\
C_{\text {non-add }} & =-6 b_{0}^{2} \int_{0}^{\infty} \mathrm{e}^{-\beta V\left(R_{12}\right)} R_{12}^{2} \int_{0}^{\infty} \mathrm{e}^{-\beta V\left(R_{13}\right)} R_{13}^{2} \\
& \int_{-1}^{1} \mathrm{e}^{-\beta V\left(R_{23}\right)}\left(\mathrm{e}^{-\beta \Delta V_{3}\left(R_{12}, R_{23}, R_{13}\right)}-1\right) \mathrm{d} X \mathrm{~d} R_{13} \mathrm{~d} R_{12}, \\
C_{\mathrm{qm}, 1} & =18 b_{0}^{2} \frac{\hbar^{2} \beta}{12 m R_{\varepsilon}^{2}} \int_{0}^{\infty} \mathrm{e}^{-\beta V\left(R_{12}\right)}\left[\frac{\mathrm{d}^{2} \beta V\left(R_{12}\right)}{\mathrm{d} R_{12}^{2}}+\frac{2}{R_{12}} \frac{\mathrm{d} \beta V\left(R_{12}\right)}{\mathrm{d} R_{12}}\right] R_{12}^{2} \\
& \left.\int_{0}^{\infty}\left(\mathrm{e}^{-\beta V\left(R_{13}\right)}-1\right) R_{13}^{2} \int_{-1}^{1}\left(\mathrm{e}^{-\beta V\left(R_{23}\right)}-1\right) \mathrm{d} X \mathrm{~d} R_{13} \mathrm{~d} R_{12}\right)
\end{aligned}
$$

with

$$
b_{0}=\frac{2}{3} \pi N_{\mathrm{A}} R_{\varepsilon}^{3}, \quad R_{23}=\sqrt{R_{12}^{2}+R_{13}^{2}-2 R_{12} R_{13} X}, \quad X=\cos \theta_{1} .
$$

Here the integration has to be performed for reduced distances.

The genuine three-body potential for the interaction between three atoms 1, 2 and 3 with the angles $\theta_{1}, \theta_{2}$ and $\theta_{3}$ between the distance vectors $\mathbf{R}_{12}, \mathbf{R}_{23}$, and $\mathbf{R}_{31}$ of the triplet is approximated by the triple-dipole potential term proposed by 
Axilrod and Teller [21,22]:

$$
\begin{aligned}
\Delta & V_{3}^{\mathrm{AT}}\left(\mathbf{R}_{1}, \mathbf{R}_{2}, \mathbf{R}_{3}\right)=\frac{C_{9}}{R_{12}^{3} R_{23}^{3} R_{31}^{3}}\left(1+3 \cos \theta_{1} \cos \theta_{2} \cos \theta_{3}\right) \\
& =\frac{C_{9}}{R_{12}^{3} R_{23}^{3} R_{31}^{3}}\left(1+\frac{3}{8} \frac{\left(R_{12}^{2}+R_{31}^{2}-R_{23}^{2}\right)\left(R_{31}^{2}+R_{23}^{2}-R_{12}^{2}\right)\left(R_{23}^{2}+R_{12}^{2}-R_{31}^{2}\right)}{R_{12}^{2} R_{23}^{2} R_{31}^{2}}\right) \\
& =\frac{C_{9}}{R_{12}^{3} R_{23}^{3} R_{31}^{3}}\left(1-3 \frac{\left(\mathbf{R}_{12} \cdot \mathbf{R}_{23}\right)\left(\mathbf{R}_{12} \cdot \mathbf{R}_{31}\right)\left(\mathbf{R}_{23} \cdot \mathbf{R}_{31}\right)}{R_{12}^{2} R_{23}^{2} R_{31}^{2}}\right) .
\end{aligned}
$$

The non-additivity coefficient of the triple-dipole term was calculated for helium by Kumar and Meath [23] to be $C_{9}=1.472$ hartree $a_{0}^{9}\left(1\right.$ hartree $\left.=3.1577465 \cdot 10^{5} \mathrm{~K}\right)$.

\subsection{Calculation of the transport properties}

The transport properties of dilute gases are formulated in different approximations of increasing order in dependence of quantum cross sections $Q^{(m)}(E)$ and quantum collision integrals $\Omega^{(m, s)}(T)$. The numbers $m$ and $s$ are connected with certain approximations of the solution of the Boltzmann equation. The quantum cross sections are given by Meeks et al. [24] in analogy to the second virial coefficient for particles with spin $s$ according to the Bose-Einstein (BE) or to the Fermi-Dirac (FD) statistics as:

$$
\begin{aligned}
& Q_{\mathrm{BE}}^{(m)}=\left[\frac{s+1}{2 s+1}\right] Q_{\mathrm{even}}^{(m)}+\left[\frac{s}{2 s+1}\right] Q_{\mathrm{odd}}^{(m)} \\
& Q_{\mathrm{FD}}^{(m)}=\left[\frac{s+1}{2 s+1}\right] Q_{\mathrm{odd}}^{(m)}+\left[\frac{s}{2 s+1}\right] Q_{\text {even }}^{(m)} .
\end{aligned}
$$

$Q_{\text {odd }}^{(m)}$ and $Q_{\text {even }}^{(m)}$ are again given in the following relationships as sums over the phase shifts $\delta_{l}$, either over only the odd $l$ values or over only the even $l$ values:

$$
\begin{aligned}
Q^{(0)} & =Q^{(1)}=Q^{(3)}=\cdots=\sum_{l}(2 l+1) \sin ^{2} \delta_{l}, \\
Q^{(2)} & =\sum_{l} \frac{(l+1)(l+2)}{(2 l+3)} \sin ^{2}\left(\delta_{l}-\delta_{l+2}\right), \\
Q^{(4)} & =\sum_{l}\left[\frac{2(l+1)(l+2)\left(2 l^{2}+6 l-3\right)}{(2 l-1)(2 l+3)(2 l+7)} \sin ^{2}\left(\delta_{l}-\delta_{l+2}\right)\right. \\
& \left.+\frac{(l+1)(l+2)(l+3)(l+4)}{(2 l+3)(2 l+5)(2 l+7)} \sin ^{2}\left(\delta_{l}-\delta_{l+4}\right)\right] .
\end{aligned}
$$

It is to point out that Eqs. (22) and (23) for even $m$ values can be applied for the Bose-Einstein and Fermi-Dirac statistics as well as for the Boltzmann statistics, whereas for the latter one the complete sums have to be used. But the simple Eq. (21) for odd $m$ values is valid only for the Bose-Einstein and Fermi-Dirac statistics, if the summation is to be performed either over the odd or over the even 
$l$ values. In the case of the Boltzmann (B) statistics more complicated relations are to be applied for the different odd $m$ values:

$$
\begin{aligned}
Q_{\mathrm{B}}^{(1)} & =\sum_{l}(l+1) \sin ^{2}\left(\delta_{l}-\delta_{l+1}\right), \\
Q_{\mathrm{B}}^{(3)} & =\sum_{l}\left[\frac{3(l+1)\left(l^{2}+2 l-1\right)}{(2 l-1)(2 l+5)} \sin ^{2}\left(\delta_{l}-\delta_{l+1}\right)\right. \\
& \left.+\frac{(l+1)(l+2)(l+3)}{(2 l+3)(2 l+5)} \sin ^{2}\left(\delta_{l}-\delta_{l+3}\right)\right] .
\end{aligned}
$$

Analogous relationships for $m=5$ and $m=6$ were given by Meeks et al. [24]. A factor $4 \pi / k^{2}$, where $k$ is again the wave number, has been dropped in this paper in all expressions for the quantum cross sections $Q^{(m)}$ compared with the relationships of Meeks et al. This factor is taken into account in the quantum collision integrals $\Omega^{(m, s)}$ defined as

$$
\Omega^{(m, s)}(T)=\frac{4 \pi \hbar^{2}}{2 \mu k_{\mathrm{B}} T(s+1)} \int_{0}^{\infty} Q^{(m)}(E), \mathrm{e}^{-\beta E}(\beta E)^{s} \mathrm{~d}(\beta E) .
$$

The viscosity and the thermal conductivity coefficients of a monatomic gas in the limit of zero density can be expressed in the $n$ th-order approximation as

$$
\begin{aligned}
{[\eta]_{n} } & =\frac{5}{16} \frac{\left(2 \pi \mu k_{\mathrm{B}} T\right)^{\frac{1}{2}}}{\Omega^{(2,2)}(T)} f_{\eta}^{(n)} \\
{[\lambda]_{n} } & =\frac{75}{64} \frac{\left(2 \pi \mu k_{\mathrm{B}}^{3} T\right)^{\frac{1}{2}}}{2 \mu \Omega^{(2,2)}(T)} f_{\lambda}^{(n)}
\end{aligned}
$$

The $\Omega^{(2,2)}$ collision integral is related to the first-order approximations for the viscosity and thermal conductivity, whereas $f_{\eta}^{(n)}$ and $f_{\lambda}^{(n)}$ represent the correction factors needed in $n$ th-order approximations of the kinetic theory. Explicit expressions up to the fifth order approximations including computer programs were prepared by Viehland et al. [25] and used for the calculations in this paper.

It is to point out that according to our calculations the effect of the fifth-order corrections to the viscosity and to the thermal conductivity compared with the fourth-order corrections is below $\pm 0.01 \%$. In this connection we refer to Figure 2 of the paper by Hurly and Moldover [1] who obtained the same results for their potential in the temperature range 10-10,000 K.

\section{Comparison with experimental data}

\subsection{Second pressure virial coefficient}

The calculation of the second virial coefficient requires to determine the possibly existing bound states. For that purpose the program Level 7.7 of LeRoy [26] was 


\section{Molecular Physics}

used and only one bound state was found to be $E_{00}=-1.64 \mathrm{mK}$ for ${ }^{4} \mathrm{He}$. This value is to be compared with $-1 \mathrm{mK}$ for the first experimental proof by Luo et al. [6]. In 2000 Grisenti et al. [8] obtained $E_{00}=-(1.1+0.3 /-0.2) \mathrm{mK}$ using diffraction experiments of a molecular beam of small helium clusters.

The comparison with the experimental data shown as absolute deviations $B_{\text {exp }}-B_{\text {cal }}$ is restricted to the best available data. For ${ }^{4} \mathrm{He}$ at low temperatures figure 1 does not only demonstrate a very good agreement for the excellent data of Berry [27] resulting from constant-volume gas thermometry, but also for the dielectric constant isotherms by Gugan and Michel [28]. The $B$ values by Kemp et al. [30] obtained also by constant-volume gas thermometry between $27 \mathrm{~K}$ and room temperature fall into line at low temperatures with the mentioned data by Berry as well as Gugan and Michel. Figure 1 also reveals a very close agreement between the $B$ values calculated with the potential model by Hurly and Mehl [2] and those obtained from the new interatomic potential of the present paper. There exists only a very small difference at the lowest temperatures.

In figure 2 absolute deviations $B_{\exp }-B_{\text {cal }}$ are presented for temperatures $T>50 \mathrm{~K}$. The figure shows an excellent agreement between the very new data by McLinden and Lösch-Will [38], measured with a high-precision two-sinker densimeter between $220 \mathrm{~K}$ and $320 \mathrm{~K}$, and the values calculated for the interatomic potential of this paper. This demonstrates the high quality of the experiments by McLinden and Lösch-Will, but also of the potential and of the statistical-mechanical calculation of the second virial coefficients. It is further illustrated that the data of Kemp et al. [30] agree at the higher temperatures with the second virial coefficients determined by Blancett et al. [33] and by Holste et al. [37]. Above room temperature the data by Schneider et al. [31,32], Waxman [34], and Kell et al. [36] are in close agreement up to about $500 \mathrm{~K}$.

Even at high temperatures above $1000 \mathrm{~K}$ the differences between the experimental data by Schneider et al. $[31,32]$ and the calculated values are not large. It is to be stressed that the calculated values are more reliable at such high temperatures.

The comparison in the case of ${ }^{3} \mathrm{He}$ is shown in Figure 3. It becomes evident that the results of four measurement series of the constant-volume gas thermometry between $1.5 \mathrm{~K}$ and $20.3 \mathrm{~K}$ performed by Matacotta et al. [40] are in close agreement with the calculated values for the interatomic helium potential. Surprisingly, the older data by Keller [39] are also reasonably consistent with the calculated values.

\subsection{Third pressure virial coefficient}

It is to point out that experimental data for the third pressure virial coefficient are not independent of the values for the second pressure virial coefficient derived from the same experiments. Hence only third pressure virial coefficients combined with second ones, which are in reasonably close agreement with the best experimental 
data and with the calculated values of the present paper, are included in the comparison. Thus the experimental data determined by McLinden and Lösch-Will [38] represent a strong criterion due to their very close agreement with regard to the second pressure virial coefficient. Figure 4 shows a comparison between experimental data and the values calculated for the new interatomic potential. This figure elucidates that an excellent agreement of the experimental data of McLinden and Lösch-Will with the calculated values is only achieved, if the third virial coefficient corresponds to the complete sum of the contributions for the pairwise additivity $C_{\text {add }}$, for the non-additivity of the three-body interatomic interactions according to Axilrod and Teller $C_{\text {non-add, }}$ and for the first-order quantum-mechanical correction $C_{\mathrm{qm}, 1}$. Good agreement is also found for the experimental data by Pfefferle et al. [41], Hoover et al. [42], Blancett et al. [33] as well as Vogl and Hall [44]. This makes evident that the calculation procedure for the third pressure virial coefficient predicts excellent values.

\subsection{Viscosity}

In principle, the initial density dependence of the experimental data for the transport properties should be considered in the discussion, since many measurements were performed at atmospheric pressure, whereas the theoretical results correspond to the limit of zero density. But this effect is comparably small $(<0.1 \%)$ for most temperatures, apart from the very low temperatures near to the normal boiling point of helium. On the other hand, the experimental uncertainty is rather high at these low temperatures so that the initial density dependence was taken into account only in one case for the thermal conductivity.

For the viscosity the situation is complicated by the fact that it is difficult to perform genuine absolute measurements of the gas viscosity with an uncertainty $< \pm 0.1 \%$, even at ambient temperature. This is demonstrated in Figure 5 for ${ }^{4} \mathrm{He}$. The measurements by Kestin and Leidenfrost [45], approved as one of the most accurate and additionally one of the few absolute measurements on gases, can only partly be considered as absolute ones. Kestin and Leidenfrost applied the theory by Newell [54], developed for absolute measurements with an oscillating-disk viscometer, and calculated first the so-called Newell's constant from the geometric dimensions of the viscometer. Then the value of Newell's constant was changed by $0.16 \%$ in order to take into account a paddle effect of the mirror used in the measurements. But for that purpose Kestin and Leidenfrost utilized a value for the viscosity of air at $20^{\circ} \mathrm{C}$ and at atmospheric pressure determined by Bearden [55] in an absolute measurement with a rotating-cylinder viscometer. Hence the genuine absolute measurement is that of Bearden. The measurements by Kestin and Nagashima [46] were analogously evaluated, but the change in Newell's constant was 0.5\%. In 1972 Kestin et al. [47] reported a best estimate of their measurements in the foregoing 


\section{Molecular Physics}

years, but with a change by nearly $+0.1 \%$ of the value at $298.15 \mathrm{~K}$ in comparison with the data by Kestin and Leidenfrost as well as Kestin and Nagashima. Hence it is to expect that all measurements which are related to these best estimates for the noble gases as well as for nitrogen should be characterized by a tendency to values increased by $+0.1 \%$. This holds for two measurement series of Vogel [50] with an all-quartz oscillating-disk viscometer which were performed in a relative manner with a Newell's constant determined from the best estimates by Kestin et al. The absolute measurements by Flynn et al. [48] and Gracki et al. [49] performed with nearly the same capillary viscometer led to values differing by $\pm 0.2 \%$. Recently, Evers et al. [51] utilized a rotating-cylinder viscometer for absolute measurements on several gases at different temperatures and pressures. Their result for helium at $293.15 \mathrm{~K}$ agrees with our calculations within $\pm 0.1 \%$ with a tendency to higher experimental data. Very recently, Berg $[52,53]$ performed highly accurate absolute measurements with a capillary viscometer only at room temperature. The experimental datum by Berg at $298.15 \mathrm{~K} \eta=(19.842 \pm 0.014) \mu \mathrm{Pas}$ (standard deviation: $2 \sigma$ ) deviates nearly $+0.1 \%$ from the calculated value $\eta=19.8262 \mu \mathrm{Pas}$ of this paper. On the other hand, the very recent calculations by Hurly and Mehl [2] with an improved interatomic potential for helium compared with that of Hurly and Moldover [1] led to a value of $\eta=(19.8245 \pm 0.004) \mu \mathrm{Pas}$ at $298.15 \mathrm{~K}$. The agreement between the calculations of Hurly and Mehl and that of the present paper in which the interatomic potential was further improved shows clearly that the uncertainty of the theoretical values is about one order of magnitude lower than that of the experiments.

The situation changes further to the disadvantage of the experiment, if the measurements are carried out away from ambient temperature. In Figure 6 experimental data at low temperatures down to $1.3 \mathrm{~K}$ and at medium temperatures up to $374 \mathrm{~K}$ are compared with the values calculated for the new potential energy curve. A close agreement within $\pm 0.5 \%$ is only found for the absolute capillary measurements of Flynn et al. [48], Gracki et al. [49] and Kao and Kobayashi [60] as well as for the absolute measurements by Evers et al. [51] with their rotatingcylinder viscometer. All other measurements are relative measurements in which the value used for the calibration plays the decisive role. Johnston and Grilly [56] (oscillating-disk viscometer) as well as Clarke and Smith [61] and Gough et al. [62] (capillary viscometers) based their measurements on reasonable values for air and nitrogen at ambient temperature resulting in deviations within $\pm 2 \%$. On the contrary, Becker et al. [57] and Becker and Misenta [58] used an old value for ${ }^{4} \mathrm{He}$ at 77.3 K from Keesom [63] for calibration in their measurements with an oscillatingcylinder viscometer so that the differences amount to about $+5 \%$. Similarly, the measurements with an oscillating-disk viscometer by Coremans et al. [59] based on an even older value for ${ }^{4} \mathrm{He}$ at $20 \mathrm{~K}$ from Kamerlingh Onnes and Weber [64] show 
positive deviations up to $5 \%$. All these results could be much better, if they would have been based on more reliable values for calibration. It is to be mentioned that in the case of measurements at atmospheric pressure a consideration of the initial density dependence of the viscosity would increase the values in the limit of zero density which means the differences would become somewhat larger. In addition, figure 6 shows only at the lowest temperatures small differences to the calculated values by Hurly and Mehl [2].

Figure 7 illustrates the comparison between the best experimental viscosity data and the calculated values at higher temperatures. For that purpose the data of the two measurement series of Vogel [50] were recalibrated at room temperature with the theoretically calculated values of ${ }^{4} \mathrm{He}$ of this report. The temperature dependence of the experimental data agrees in an excellent manner with the calculated values at all other temperatures up to $650 \mathrm{~K}$. The measurements by Vogel with his all-quartz oscillating-disk viscometer represent the best experiments in this temperature range.

Although the values of the best estimate by Kestin et al. [47] and the experimental data of a further paper by Kestin et al. [65] were not recalibrated, Figure 7 reveals a systematic trend in the data by Kestin et al. to higher values with increasing temperature. But this tendency is well-known for all the measurements by Kestin and his co-workers with the oscillating-disk viscometer developed by Di Pippo et al. [68]. These systematic deviations are a consequence of a temperature measurement error with thermocouples extensively discussed by Vogel et al. [69] and are still relatively small for helium due to the large thermal conductivity coefficient compared with those of other common gases. The relative measurements of Guevara et al. [66] and of Dawe and Smith [67] with capillary viscometers based on a reasonable calibration at room temperature make obvious that they are influenced by systematic errors and that the theoretical calculation is distinctly superior to the experiment at these high temperatures.

Figure 8 displays the deviations of the experimental viscosity data by Becker et al. [57] and Becker and Misenta [58] from the theoretically calculated values for ${ }^{3} \mathrm{He}$. These differences are not too large with respect to the uncertainty of $\pm 5 \%$ estimated by those authors.

\subsection{Thermal conductivity}

Accurate measurements of the thermal conductivity are difficult to carry out due to different experimental problems. Results for ${ }^{4} \mathrm{He}$ near to room temperature obtained with the transient hot-wire technique, the most accurate method for determining thermal conductivity coefficients, are compared in Figure 9 with the values theoretically calculated. This comparison is a further stringent test of the new potential and of the correct application of the kinetic theory including the 


\section{Molecular Physics}

quantum-mechanical effects. The experimental data by Kestin et al. [71] and Assael et al. [72] as well as by Johns et al. [74] differ from the calculated values by $< \pm 0.1 \%$ and $< \pm 0.2 \%$, less than the uncertainties estimated by those authors themselves $( \pm 0.3 \%)$. The deviation of the first experiment with this method by Haarman [70] is only somewhat larger, whereas that of Mustafa et al. [73] is distinctly increased.

It is to note that the differences between the calculated values by Hurly and Mehl [2] and those of the present paper are too small to become obvious in figures $9-11$.

The experimental thermal conductivity data for ${ }^{4} \mathrm{He}$ below ambient temperature are compared in Figure 10 with the calculated values. It becomes evident that there exists an excellent agreement for the experimental data of Acton and Kellner [81] obtained between $3.3 \mathrm{~K}$ and $20 \mathrm{~K}$ with a parallel-plate apparatus. It is to be stressed that we extrapolated the experimental density series of Acton and Kellner to the limit of zero density for this comparison. But the experimental data between $2.08 \mathrm{~K}$ and $3.95 \mathrm{~K}$ by Kerrisk and Keller [77] resulting also from parallel-plate measurements show large positive differences. These values were not corrected, since the measurements were carried out only at one pressure of about 10 Torr. The effect of the density dependence is distinctly smaller than the deviations. The experimental data between $7.7 \mathrm{~K}$ and $273 \mathrm{~K}$ by Popov and Zarev [82] using the concentric-cylinder method show similar positive differences to the theoretically calculated values with decreasing temperature. These data could also not be corrected with respect to the initial density dependence, since details about the pressure or density of the measurements are missing. The experimental data by Zarev et al. [83] (concentric-cylinder method) and by Roder [78,79] (parallel-plate technique) are characterized by comparably small deviations from the calculated values.

Figure 11 illustrates the comparison above ambient temperature. The measurement of Johns et al. [74] at $378 \mathrm{~K}$ agrees again within $\pm 0.1 \%$ with the calculated value. Furthermore, the results of the measurements of Haarman [70] between $328 \mathrm{~K}$ and $468 \mathrm{~K}$ deviate in average by $-0.4 \%$, but show nearly the same temperature dependence as the calculated values. The differences of the measurements by Mustafa et al. [73] cannot be explained with respect to the much valued transient hot-wire technique. The experimental data by Vargaftik and Zhimina [84] (common hot-wire technique) and by Le Neindre et al. [85] (concentric-cylinder method) are characterized by not too large deviations from the calculated values, but do not allow any test of the potential and of the kinetic theory.

Figure 12 shows for ${ }^{3} \mathrm{He}$ the deviations of the experimental thermal conductivity data by Kerrisk and Keller [77] between $1.5 \mathrm{~K}$ and $3.95 \mathrm{~K}$ and by Zarev et al. [83] between $79 \mathrm{~K}$ and $276 \mathrm{~K}$ from the theoretical values. The differences correspond 
approximately to those for ${ }^{4} \mathrm{He}$.

\section{Conclusion}

A new potential function for helium [3] was used for the quantum-mechanical calculation of the second and third pressure virial, of the viscosity and of the thermal conductivity coefficients for ${ }^{4} \mathrm{He}$ and ${ }^{3} \mathrm{He}$ in the range from $1 \mathrm{~K}$ to $10,000 \mathrm{~K}$. The extensive comparison with experimental data as well as with recent calculations by Hurly and Mehl [2] using a potential function obtained from a fit to various ab initio calculations from the literature makes evident that the theoretically calculated values of the thermophysical properties are characterized by uncertainties superior to any experiment. In the case of the second pressure virial coefficient the differences between the results obtained by our potential and the potential of Hurly and Mehl give an estimate of the uncertainties of this property. Values of the third pressure virial coefficient calculated classically including a non-additive contribution according to the Axilrod-Teller potential model and a quantum-mechanical correction are in excellent agreement with very recent experimental data by McLinden and Lösch-Will [38]. For both viscosity and thermal conductivity the relative differences between the results obtained from the two potentials do not exceed $\pm 0.01 \%$ for temperatures above $15 \mathrm{~K}$ and increase to $\pm 0.13 \%$ at $1 \mathrm{~K}$. This shows that the transport properties are practically insensitive to small changes in the potential function. To get reliable error bars we stress that contributions from the kinetic theory beyond the fifth-order approximation are distinctly smaller than $\pm 0.01 \%$ (see Figure 2 of Reference [1]). In addition, all digits of the calculated values given by Hurly and Mehl for viscosity and thermal conductivity could be reproduced when applying their potential function and using our computer code. Hence the uncertainties in viscosity and thermal conductivity should be primarily due to the errors in the potential. Since our potential is more accurate than the one of Hurly and Mehl, we would suggest $\pm 0.02 \%$ as a conservative estimate of the relative uncertainties for both properties down to $15 \mathrm{~K}$. At temperatures lower than $15 \mathrm{~K}$ the uncertainty increases to $\pm 0.2 \%$ at $1 \mathrm{~K}$, but is still far below any experimental uncertainty. The theoretical values for all calculated thermophysical properties can safely be recommended as standard values for ${ }^{3} \mathrm{He}$ and ${ }^{4} \mathrm{He}$ in the temperature range from $1 \mathrm{~K}$ to $10,000 \mathrm{~K}$ apart from the third pressure virial coefficient, for which the quantum correction is certainly not applicable at temperatures below $20 \mathrm{~K}$. The calculated values are listed in the Appendix.

\section{Acknowledgments}

We wish to thank Larry Viehland for providing his Fortran code. 


\section{Molecular Physics}

\section{References}

[1] J. J. Hurly and M. R. Moldover, J. Res. Natl. Inst. Stand. Technol., 105, 667 (2000).

[2] J. J. Hurly and J. B. Mehl, J. Res. Natl. Inst. Stand. Technol., 112, 75 (2007).

[3] R. Hellmann, E. Bich and E. Vogel, Mol. Phys., (submitted).

[4] K. T. Tang and J. P. Toennies, J. Chem. Phys., 80, 3726 (1984).

[5] H. B. G. Casimir and D. Polder, Phys. Rev., 73, 360 (1948).

[6] F. Luo, G. C. McBane, G. Kim, C. F. Giese and W. R. Gentry, J. Chem. Phys., 98, 3564 (1993).

[7] W. Schöllkopf and J. P. Toennies, Science, 266, 1345 (1994).

[8] R. E. Grisenti, W. Schöllkopf, J. P. Toennies, G. C. Hegerfeldt, T. Köhler and M. Stoll, Phys. Rev. Lett., 85, 2284 (2000).

[9] M. J. Jamieson, G. W. F. Drake and A. Dalgarno, Phys. Rev. A., 51, 3358 (1995).

[10] A. R. Janzen and R. A. Aziz, J. Chem. Phys., 107, 914 (1997).

[11] M.-K. Chen and K. T. Chung, Phys. Rev. A, 53, 1439 (1996).

[12] N. C. Handy and A. M. Lee, Chem. Phys. Lett., 252, 425 (1996).

[13] W. Kutzelnigg, Mol. Phys., 90, 909 (1997).

[14] G. T. McConville and J. J. Hurly, Metrologia, (1991) 28, 375 (1991).

[15] R. J. LeRoy, Computer Program for Calculating Phase Shifts and Time Delays for Scattering on a Spherical Potential, University of Waterloo, Chemical Physics Research Report CP-107R, Waterloo, Ontario, Canada.

[16] J. J. Hurly, G. T. McConville and W. L. Taylor, Algorithms and Fortran Programs to Calculate Quantum Collision Integrals for Realistic Intermolecular Potentials, MLM-3635, Miamisburg, EG\&GMAT (1990).

[17] M. Abramowitz and I. A. Stegun, Handbook of Mathematical Functions, Dover, New York, 365 (1972).

[18] M. E. Boyd, S. Y. Larsen and J. E. Kilpatrick, J. Chem. Phys., 50, 4034 (1969).

[19] S. Kim and D. Henderson, Proc. Nat. Acad. Sci., Wash., 55, 705 (1966).

[20] K. Lucas, Angewandte Statistische Thermodynamik, Springer, Berlin (1986).

[21] B. M. Axilrod and E. Teller, J. Chem. Phys., 11, 299 (1943).

[22] B. M. Axilrod, J. Chem. Phys., 19, 719 (1951).

[23] A. Kumar and W. J. Meath, Mol. Phys., 54, 823 (1985).

[24] F. R. Meeks, T. J. Cleland, K. E. Hutchinson and W. L. Taylor, J. Chem. Phys., 100, 3813 (1994).

[25] L. A. Viehland, A. R. Janzen and R. A. Aziz, J. Chem. Phys., 102, 5444 (1995).

[26] R. J. LeRoy, LEVEL 7.7. A Computer Program for Solving the Radial Schrödinger Equation for Bound and Quasibound Levels, University of Waterloo, Chemical Physics Research Report CP-661, Waterloo, Ontario, Canada.

[27] K. H. Berry, Metrologia, 15, 89 (1979).

[28] D. Gugan and G. W. Michel, Metrologia, 16, 149 (1980).

[29] R. A. Aziz, Mol. Phys., 61, 1487 (1987).

[30] R. C. Kemp, W. R. G. Kemp and L. M. Besley, Metrologia, 23, 61 (1986/87).

[31] W. G. Schneider and J. A. H. Duffie, J. Chem. Phys., 17, 751 (1949).

[32] J. L. Yntema and W. G. Schneider, J. Chem. Phys., 18, 641 (1950).

[33] A. L. Blancett, K. R. Hall and F. B. Canfield, Physica, 47, 75 (1970).

[34] M. Waxman as cited by L. A. Guildner and R. E. Edsinger, J. Res. Natl. Bur. Stand., 80, 703 (1976).

[35] M. Waxman and H. Davis, J. Res. Natl. Bur. Stand., 83, 415 (1978).

[36] G. S. Kell, G. E. McLaurin and E. Whalley, J. Chem. Phys., 68, 2199 (1978).

[37] J. C. Holste, M. Q. Watson, M. T. Bellomy, P. T. Eubank and K. R. Hall, AIChE J., 26, 954 (1980).

[38] M. O. McLinden and C. Lösch-Will, J. Chem. Thermodyn., 39, 507 (2007).

[39] W. E. Keller, Phys. Rev., 98, 1571 (1955).

[40] F. C. Matacotta, G. T. McConville, P. P. M. Steur and M. Durieux, Metrologia, 24, 61 (1987).

[41] W. C. Pfefferle, J. A. Goff and J. G. Miller, J. Chem. Phys., 23, 509 (1955).

[42] A. E. Hoover, F. B. Canfield, R. Kobayashi and T. W. Leland, Jr., J. Chem. Eng. Data, 9, 568 (1964).

[43] J. A. Provine and F. B. Canfield, Physica, 52, 79 (1971).

[44] W. F. Vogl and K. R. Hall, Physica, 59, 529 (1972).

[45] J. Kestin and W. Leidenfrost, Physica, 25, 1033 (1959).

[46] J. Kestin and A. Nagashima, J. Chem. Phys., 40, 3648 (1964).

[47] J. Kestin, S. T. Ro and W. A. Wakeham, J. Chem. Phys., 56, 4119 (1972).

[48] G. P. Flynn, R. V. Hanks, N. A. Lemaire and J. Ross, J. Chem. Phys., 38, 154 (1963).

[49] J. A. Gracki, G. P. Flynn and J. Ross, J. Chem. Phys., 51, 3856 (1969).

[50] E. Vogel, Ber. Bunsenges. Phys. Chem., 88, 997 (1984). 


\section{Helium2a-MolPhys \\ Molecular Physics}

[51] C. Evers, H. W. Lösch and W. Wagner, Int. J. Thermophys., 23, 1411 (2002).

[52] R. F. Berg, Metrologia, 42, 11 (2005).

[53] R. F. Berg, Metrologia, 43, 183 (2006).

[54] G. F. Newell, Z. Ang. Math. Phys., 10, 160 (1959).

[55] J. A. Bearden, Phys. Rev., 56, 1023 (1939).

[56] H. L. Johnston and E. R. Grilly, J. Phys. Chem., 46, 948 (1942).

[57] E. W. Becker, R. Misenta and F. Schmeissner, Z. Phys., 137, 126 (1954).

[58] E. W. Becker and R. Misenta, Z. Phys., 140, 535 (1955).

[59] J. M. J. Coremans, A. van Itterbeek, J. J. M. Beenakker, H. F. P. Knaap and P. Zandbergen, Physica, 24, 557 (1958).

[60] J. T. F. Kao and R. Kobayashi, J. Chem. Phys., 47, 2836 (1967).

[61] A. G. Clarke and E. B. Smith, J. Chem. Phys., 51, 4156 (1969).

[62] D. W. Gough, G. P. Matthews and E. B. Smith, J. Chem. Soc., Faraday Trans. I, 72, 645 (1976).

[63] W. H. Keesom, Helium, Elsevier, Amsterdam, 1942, 107.

[64] H. Kamerlingh Onnes and S. Weber Vers. Kon. Acad. Wetenschapen, Amsterdam, 21, 1385 (1913).

[65] J. Kestin, S. T. Ro and W. A. Wakeham, J. Chem. Phys., 56, 5837 (1972).

[66] F. A. Guevara, B. B. McInteer and W. E. Wageman, Phys. Fluids, 12, 2493 (1969).

[67] R. A. Dawe and E. B. Smith, J. Chem. Phys., 52, 693 (1970).

[68] R. Di Pippo, J. Kestin and J. H. Whitelaw, Physica, 32, 2064 (1966).

[69] E. Vogel, C. Küchenmeister, E. Bich and A. Laesecke J. Phys. Chem. Ref. Data, 27, 947 (1998).

[70] J. W. Haarman, Amer. Inst. Phys. Conf. Proc., 11, 193 (1973).

[71] J. Kestin, R. Paul, A. A. Clifford and W. A. Wakeham, Physica A, 100, 349 (1980).

[72] M. J. Assael, M. Dix, A. Lucas and W. A. Wakeham, J. Chem. Soc., Faraday Trans. I, 77, 439 (1981).

[73] M. Mustafa, M. Ross, R. D. Trengove, W. A. Wakeham and M. Zalaf, Physica A, 141, 233 (1987).

[74] A. I. Johns, A. C. Scott, J. T. R. Watson, D. Ferguson and A. A. Clifford, Phil. Trans. Roy. Soc., London, 325, 295 (1988).

[75] J. B. Ubbink and W. J. de Haas, Physica, 10, 465 (1943).

[76] I. F. Golubev and I. B. Shpagina, Gasovaya Promyshlennost, 11, 40 (1966).

[77] J. F. Kerrisk and W. E. Keller, Phys. Rev., 177, 341 (1969).

[78] H. M. Roder, Thermodynamiksymposium, Heidelberg, Germany 1967.

[79] H. M. Roder, NBS Laboratory Note, Project No. 2750426, Jan. 29, 1971.

[80] A. G. Shashkov, N. A. Nesterov, V. M. Sudnik and V. I. Alejnikova, Inzh. Fiz. Zh., 30, 671 (1976).

[81] A. Acton and K. Kellner, Physica B, 90, 192 (1977).

[82] V. N. Popov and V. V. Zarev, Trudy Mosk. Energ. Inst., 532, 12 (1981).

[83] V. V. Zarev, D. N. Nagorov, V. A. Nikonorov and V. N. Popov, Moskvus Sb. Trudy, Mosk. Energ. Inst., 72, 185 (1985).

[84] N. B. Vargaftik and N. Ch. Zhimina, Atomnaya Energiya, 19, 300 (1965).

[85] B. LeNeindre, R. Tufeu, P. Bury, P. Johannin and B. Vodar, Proc. 8th Conf. Thermal Cond. (1968), Plenum, New York, 75 (1969).

[86] F. M. Faubert and G. S. Springer, J. Chem. Phys., 58, 4080 (1973).

[87] E. I. Martchenko and A. G. Shashkov, Inzh. Fiz. Zh., 26, 1089 (1974).

[88] B. J. Jody, S. C. Saxena, V. P. S. Nain and R. A. Aziz, Chem. Phys., 22, 53 (1977). 


\section{September 27, $2007 \quad$ 13:13 $\quad$ Molecular Physics $\quad$ Helium2a-MolPhys \\ Molecular Physics}

Table 1. Potential parameters $\left(\varepsilon / k_{\mathrm{B}}, R_{\varepsilon}\right.$, and $\sigma$ for the retarded potential).

\begin{tabular}{lr}
\hline$A(\mathrm{~K})$ & $0.307092338615 E+07$ \\
$a_{1}\left(a_{0}^{-1}\right)$ & $-0.201651289932 E+01$ \\
$a_{-1}\left(a_{0}\right)$ & $-0.431646276045 E+00$ \\
$a_{2}\left(a_{0}^{-2}\right)$ & $-0.459521265125 E-01$ \\
$a_{-2}\left(a_{0}^{2}\right)$ & $0.138539045980 E+00$ \\
$d_{1}$ & $0.167127323768 E-02$ \\
$d_{2}\left(a_{0}^{-1}\right)$ & $0.178284243205 E+01$ \\
$d_{3}$ & $0.176635702255 E+01$ \\
$b\left(a_{0}^{-1}\right)$ & $0.203625105759 E+01$ \\
$C_{6}\left(\mathrm{~K} a_{0}^{6}\right)$ & $0.4616213781 E+06$ \\
$C_{8}\left(\mathrm{~K} a_{0}^{8}\right)$ & $0.4460565781 E+07$ \\
$C_{10}\left(\mathrm{~K} a_{0}^{10}\right)$ & $0.5803352873 E+08$ \\
$C_{12}\left(\mathrm{~K} a_{0}^{12}\right)$ & $0.1031677697 E+10$ \\
$C_{14}\left(\mathrm{~K} a_{0}^{14}\right)$ & $0.2415716766 E+11$ \\
$C_{16}\left(\mathrm{~K} a_{0}^{16}\right)$ & $0.7191492488 E+12$ \\
$\varepsilon / k_{\mathrm{B}}(\mathrm{K})$ & 10.997898 \\
$R_{\varepsilon}\left(a_{0}\right)$ & 5.608068 \\
$\sigma\left(a_{0}\right)$ & 4.990672 \\
\hline
\end{tabular}




\section{September 27,2007
Page $\mathbf{1 9}$ of $\mathbf{4 7}$ \\ Molecular Physics}

II. Thermophysical standard values for low-density helium

Table 2. Number of calculated phase shifts for some reduced energies

\begin{tabular}{ccc}
\hline$E^{*}$ & Total number & QM [Eq. (3)-(6)] \\
\hline 0. & 1 & 1 \\
$1 . \times 10^{-5}$ & 4 & 4 \\
$1 . \times 10^{-2}$ & 13 & 6 \\
$1 . \times 10^{-1}$ & 34 & 12 \\
1. & 87 & 24 \\
10. & 454 & 42 \\
100. & 454 & 70 \\
$1,000$. & 454 & 106 \\
$10,000$. & 618 & 196 \\
$24,000$. & 790 & 230 \\
$25,000$. & 809 & 0 \\
\hline
\end{tabular}

16

17

18

19

20 
Fig. 1 Deviations of experimental and calculated second pressure virial coefficients from values calculated with the new interatomic potential for ${ }^{4} \mathrm{He}$ at low temperatures. Experimental data: ○ Berry [27]; $\triangle$ Gugan and Michel [28], smoothed data from Aziz [29]; - Kemp et al. [30]. Calculated values: - - - Hurly and Mehl [2].

Fig. 2 Deviations of experimental and calculated second pressure virial coefficients from values calculated with the new interatomic potential for ${ }^{4} \mathrm{He}$ at medium and higher temperatures. Experimental data:

Kemp et al. [30]; $\Delta$ Schneider and Duffie [31] as well as Yntema and Schneider [32]; • Blancett et al. [33]; $\nabla$ Waxman [34]; $\mathbf{\nabla}$ Waxman and Davis [35]; $\triangle$ Kell et al. [36]; $\square$ Holste et al. [37]; ○ McLinden and Lösch-Will [38]. Calculated values: - - - Hurly and Mehl [2].

Fig. 3 Deviations of experimental and calculated second pressure virial coefficients from values calculated with the new interatomic potential for ${ }^{3}$ He. Experimental data: $\circ$ Keller [39]; $\triangle, \diamond, \nabla, \square$, run 1 to 4, Matacotta et al. [40]. Calculated values: - - - Hurly and Mehl [2].

Fig. 4 Comparison of experimental data and and of values for the third pressure virial coefficient derived from the new interatomic potential for ${ }^{4}$ He. Experimental data: — Pfefferle et al. [41]; $\boldsymbol{\Delta}$ Hoover et al. [42]; • Blancett et al. [33]; $\nabla$ Provine and Canfield [43]; $\mathbf{\nabla}$ Vogl and Hall [44]; $\triangle$ Kell et al. [36]; ○ McLinden and Lösch-Will [38]. Calculated values: - - - classical contribution $C_{\text {add }},-\cdot-\cdot-\cdot$ classical and nonadditivity contributions $C_{\text {add }}+C_{\text {non-add }},-$ sum of classical and non-additivity contributions and of the first-order quantum correction $C_{\text {add }}+C_{\text {non-add }}+C_{\mathrm{qm}, 1}$.

Fig. 5 Deviations of experimental and calculated viscosity coefficients from values calculated with the new interatomic potential for ${ }^{4} \mathrm{He}$ at room temperature. Experimental data: - Kestin and Leidenfrost [45]; ○ Kestin and Nagashima [46]; $\odot$ Kestin et al. [47]; - Flynn et al. [48]; $\square$ Gracki et al. [49]; $\Delta$ Vogel [50], 1st series of measurements; $\triangle$ Vogel [50], 2nd series of measurements; $₫$ Evers et al. [51]; $\nabla$ Berg $[52,53]$. Calculated values: - - - Hurly and Mehl [2].

Fig. 6 Deviations of experimental and calculated viscosity coeefficients from values calculated with the new interatomic potential for ${ }^{4} \mathrm{He}$ at low and medium temperatures. Experimental data: $\star$ Johnston and Grilly [56];

- Becker et al. [57]; ○ Becker and Misenta [58]; $\triangle$ Coremans et al. [59]; - Flynn et al. [48]; $\square$ Gracki et al. [49]; $\diamond$ Kao and Kobayashi [60]; $\nabla$ Clarke and Smith [61]; $\nabla$ Gough et al. [62]; $\square$ Evers et al. [51]. Calculated values: - - - Hurly and Mehl [2].

Fig. 7 Deviations of experimental and calculated viscosity coefficients from 
values calculated with the new interatomic potential for ${ }^{4} \mathrm{He}$ at higher temperatures. Experimental data: - Kestin et al. [47]; ○ Kestin et al. [65]; $\boldsymbol{\nabla}$ Guevara et al. [66]; $\square$ Dawe and Smith [67]; $\boldsymbol{\Delta}$ Vogel [50], 1st series of measurements recalibrated; $\triangle$ Vogel [50], 2nd series of measurements recalibrated. Calculated values: - - - Hurly and Mehl [2].

Fig. 8 Deviations of experimental and calculated viscosity coefficients from values calculated with the new interatomic potential for ${ }^{3} \mathrm{He}$. Experimental data: • Becker et al. [57]; ○ Becker and Misenta [58]. Calculated values: - - - Hurly and Mehl [2].

Fig. 9 Deviations of experimental and calculated thermal conductivity coefficients from values calculated with the new interatomic potential for ${ }^{4} \mathrm{He}$ at room temperature. Experimental data: • Haarman [70]; $\diamond$ Kestin et al. [71]; - Assael et al. [72]; $\boldsymbol{\nabla}$ Mustafa et al. [73]; $\boldsymbol{\Delta}$ Johns et al. [74]. Calculated values: - - - Hurly and Mehl [2].

Fig. 10 Deviations of experimental and calculated thermal conductivity coefficients from values calculated with the new interatomic potential for ${ }^{4} \mathrm{He}$ at low and medium temperatures. Experimental data: $\circ$ Ubbink and de Haas [75]; $\triangle$ Golubev and Shpagina [76]; • Kerrisk and Keller [77]; $\Delta$ Roder [78, 79]; $\square$ Shashkov et al. [80]; - Acton and Kellner [81]; $\nabla$ Popov and Zarev [82]; $\nabla$ Zarev et al. [83]. Calculated values: - - Hurly and Mehl [2].

Fig. 11 Deviations of experimental and calculated thermal conductivity coefficients from values calculated with the new interatomic potential for ${ }^{4} \mathrm{He}$ at higher temperatures. Experimental data: $\triangle$ Vargaftik and Zhimina [84]; $\square$ LeNeindre et al. [85]; • Haarman [70]; $\nabla$ Faubert and Springer [86]; ○ Martchenko and Shashkov [87]; $\diamond$ Jody et al. [88]; Mustafa et al. [73]; $\boldsymbol{\Delta}$ Johns et al. [74]. Calculated values: - - - Hurly and Mehl [2].

Fig. 12 Deviations of experimental and calculated thermal conductivity coefficients from values calculated with the new interatomic potential for ${ }^{3}$ He. Experimental data: • Kerrisk and Keller [77]; $\nabla$ Zarev et al. [83]. Calculated values: - - - Hurly and Mehl [2]. 


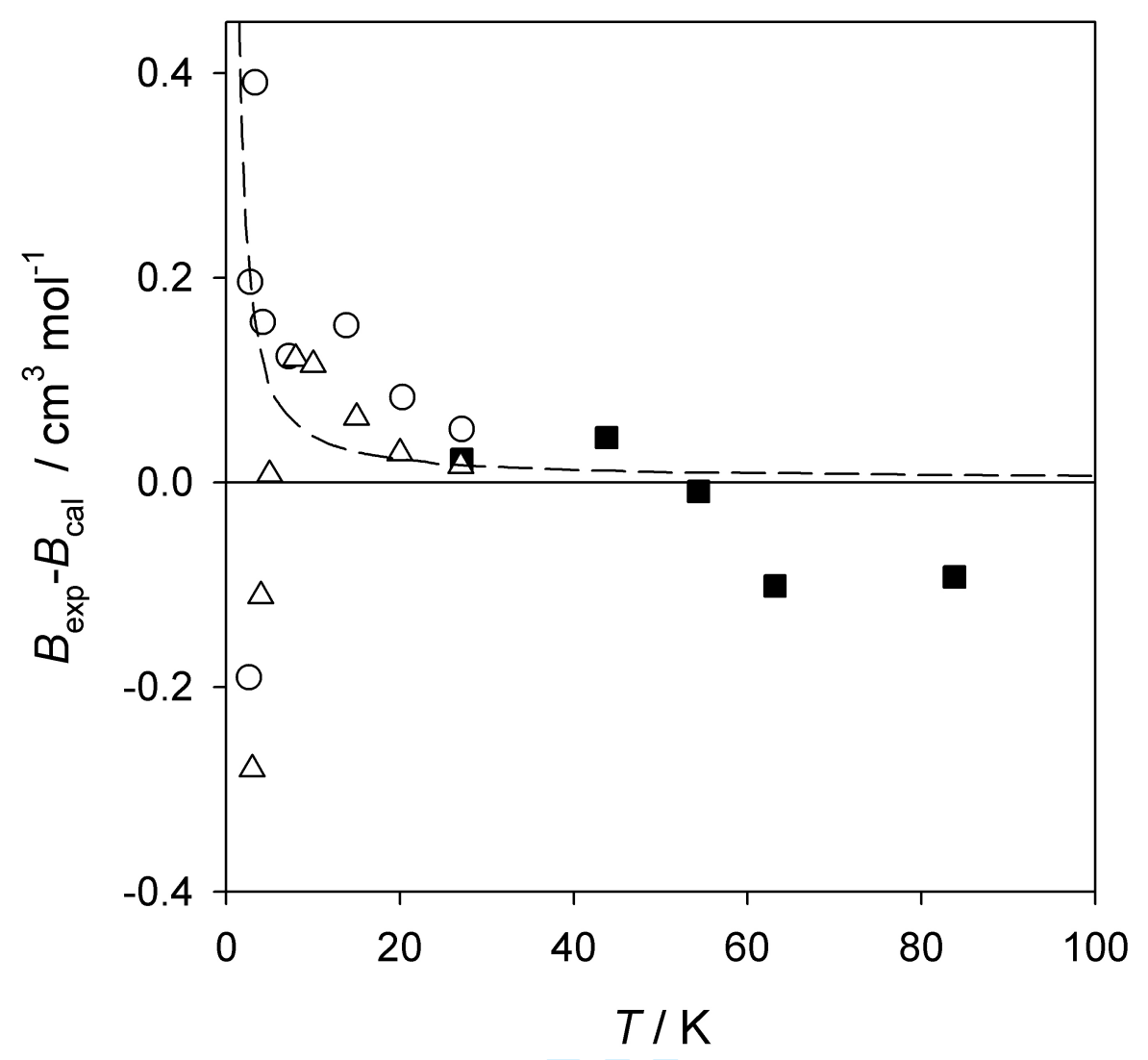

Figure 1. Deviations of experimental and calculated second pressure virial coefficients from values calculated with the new interatomic potential for ${ }^{4} \mathrm{He}$ at low temperatures. Experimental data: 0 Berry [27]; $\triangle$ Gugan and Michel [28], smoothed data from Aziz [29]; $\boldsymbol{a}$ Kemp et al. [30]. Calculated values: - - - Hurly and Mehl [2]. 


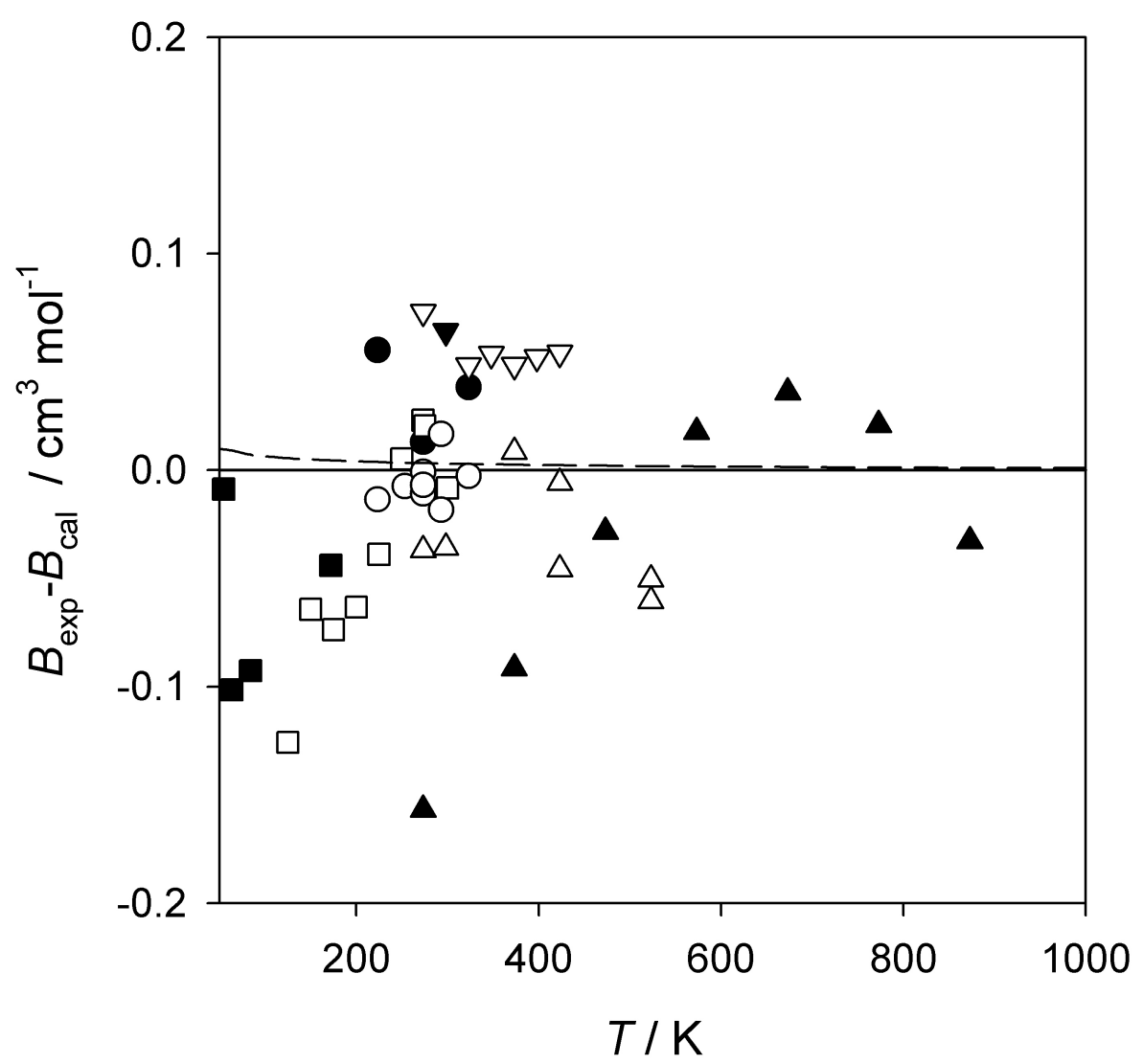

Figure 2. Deviations of experimental and calculated second pressure virial coefficients from values calculated with the new interatomic potential for ${ }^{4} \mathrm{He}$ at medium and higher temperatures. Experimental data: $\boldsymbol{\square}$ Kemp et al. [30]; $\boldsymbol{\Delta}$ Schneider and Duffie [31] as well as Yntema and Schneider [32]; $\bullet$ Blancett et al. [33]; $\nabla$ Waxman [34]; $\mathbf{V}$ Waxman and Davis [35]; $\triangle$ Kell et al. [36]; $\square$ Holste et al. [37]; ○ McLinden and Lösch-Will [38]. Calculated values: - - Hurly and Mehl [2]. 
Figure 3. Deviations of experimental and calculated second pressure virial coefficients from values calculated with the new interatomic potential for ${ }^{3}$ He. Experimental data: $\odot$ Keller [39]; $\Delta, \diamond, \nabla, \square$, run 1 to 4, Matacotta et al. [40]. Calculated values: - - Hurly and Mehl [2]. 


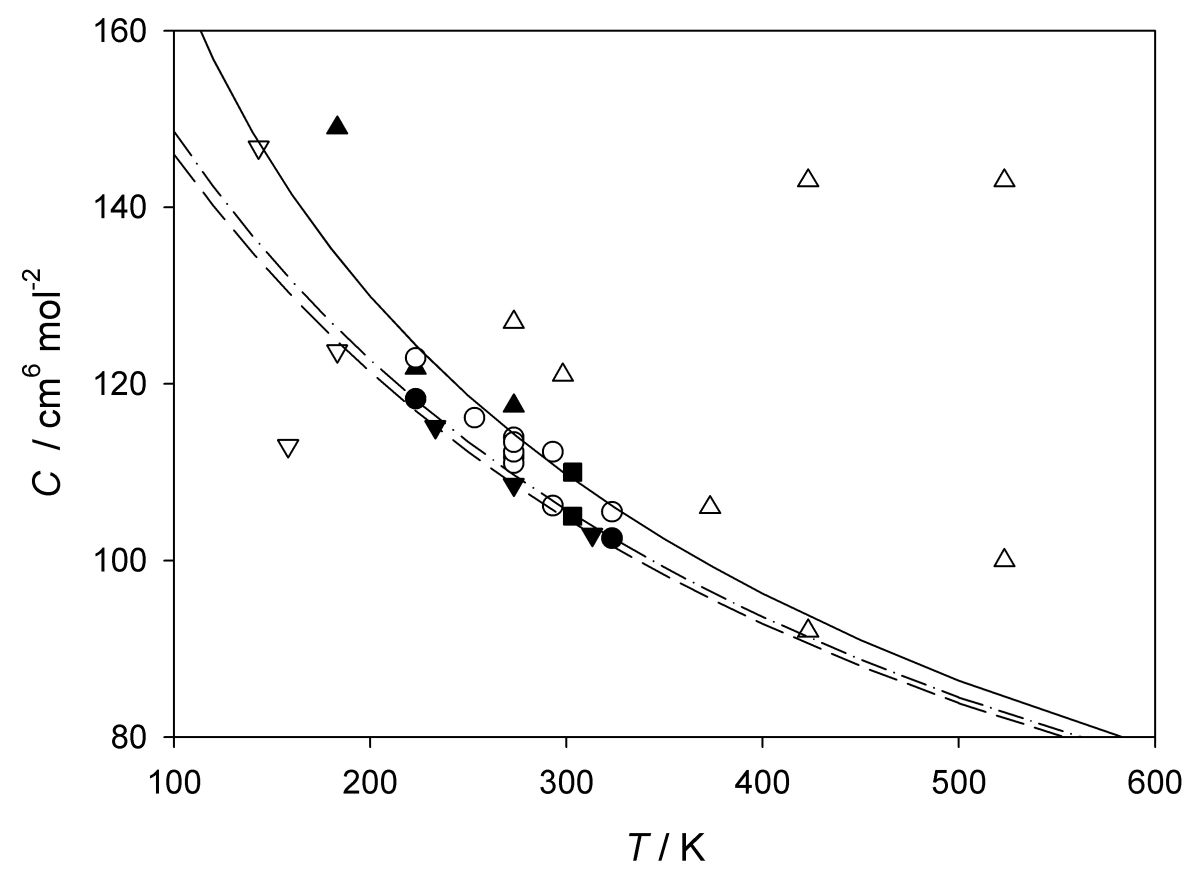

Figure 4. Comparison of experimental data and and of values for the third pressure virial coefficient derived from the new interatomic potential for ${ }^{4} \mathrm{He}$. Experimental data: $\boldsymbol{\text { 口 }}$ Pefferle et al. [41]; $\boldsymbol{\Delta}$ Hoover et al. [42]; • Blancett et al. [33]; $\nabla$ Provine and Canfield [43]; $\boldsymbol{\nabla}$ Vogl and Hall [44]; $\triangle$ Kell et al. [36]; $\bigcirc$ McLinden and Lösch-Will [38]. Calculated values: --- classical contribution $C_{\text {add }},-\cdot-\cdot-\cdot$ classical and non-additivity contributions $C_{\text {add }}+C_{\text {non-add }},-$ sum of classical and non-additivity contributions and of the first-order quantum correction $C_{\mathrm{add}}+C_{\mathrm{non}-\text { add }}+C_{\mathrm{qm}, 1}$. 
1

2

3

4

5

6

7

8

9

10

11

12

13

14

15

16

17

18

19

20

21

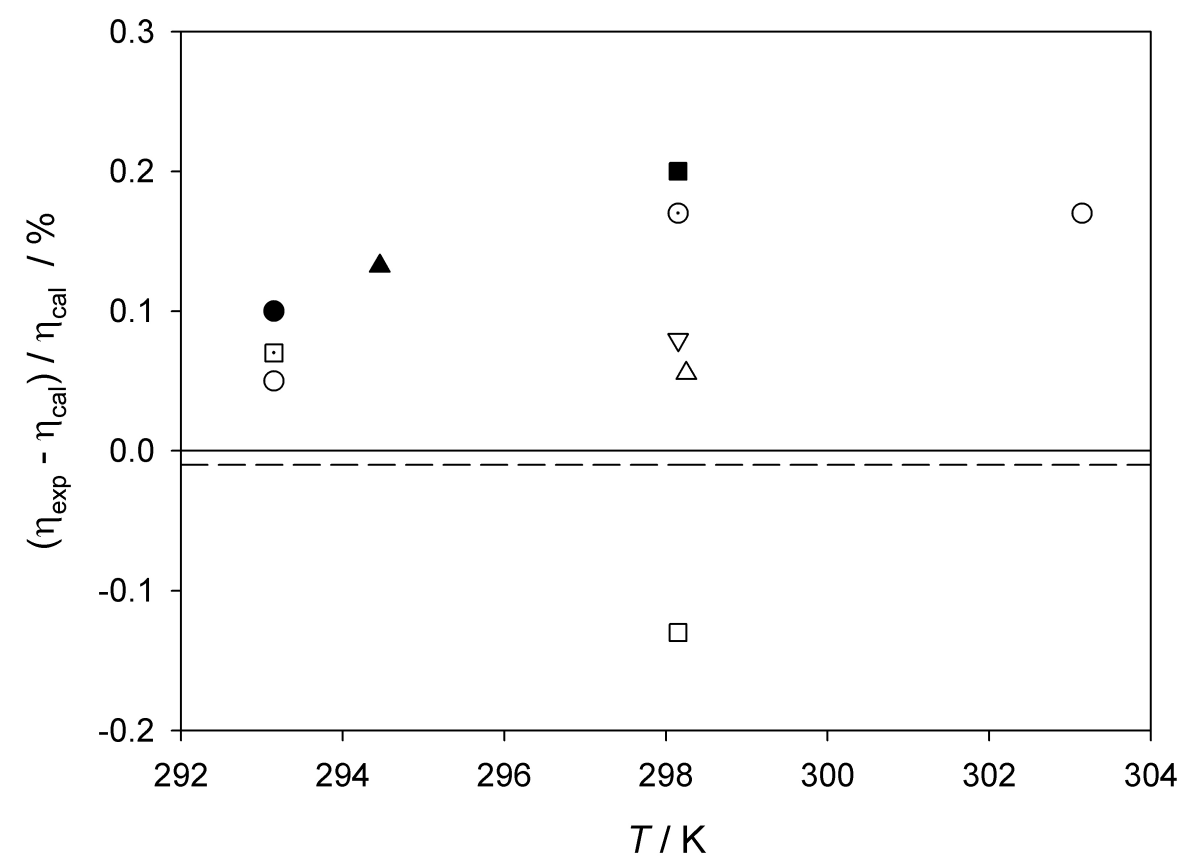

Figure 5. Deviations of experimental and calculated viscosity coefficients from values calculated with the new interatomic potential for ${ }^{4} \mathrm{He}$ at room temperature. Experimental data: - Kestin and Leidenfrost [45]; ○ Kestin and Nagashima [46]; ๑ Kestin et al. [47]; - Flynn et al. [48]; $\square$ Gracki et al. [49]; $\Delta$ Vogel [50], 1st series of measurements; $\triangle$ Vogel [50], 2nd series of measurements; $\square$ Evers et al. [51]; $\nabla$ Berg [52,53]. Calculated values: - - Hurly and Mehl [2]. 


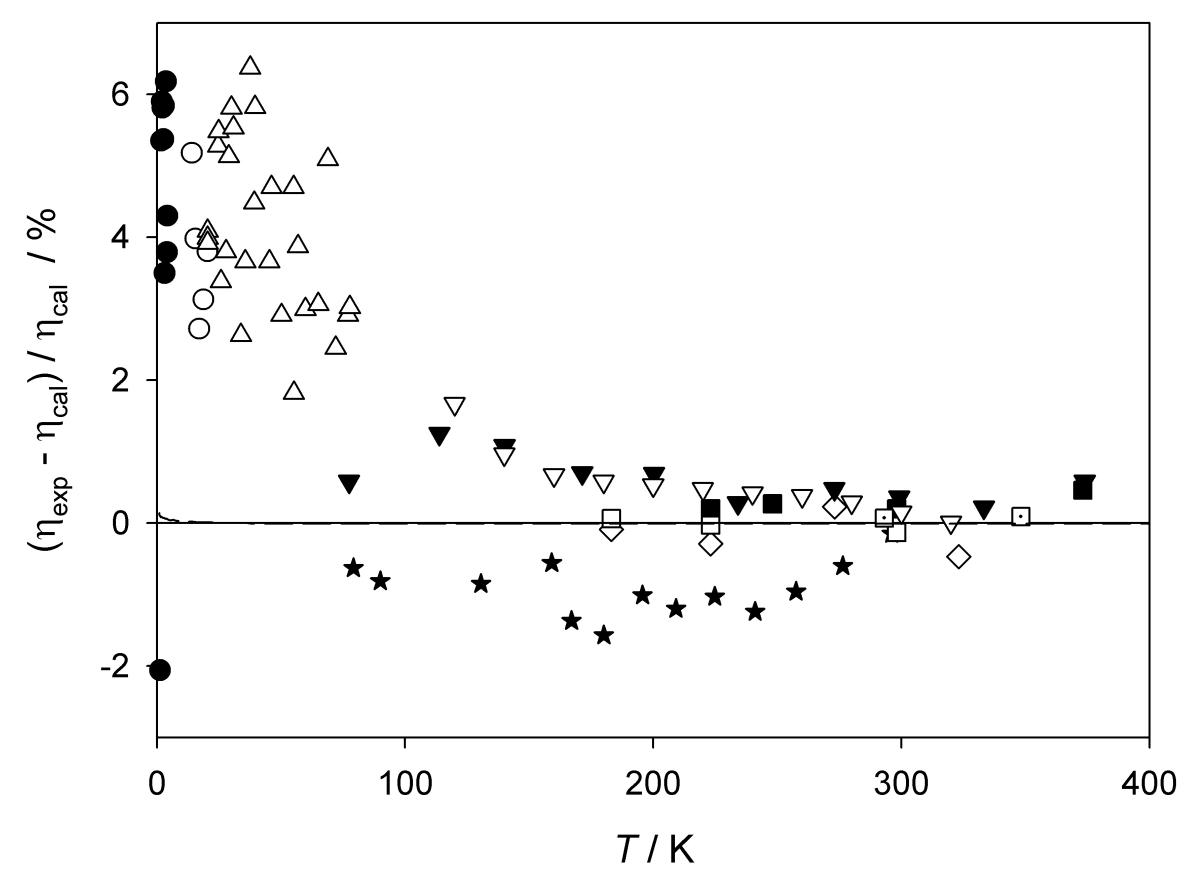

Figure 6. Deviations of experimental and calculated viscosity coefficients from values calculated with the new interatomic potential for ${ }^{4} \mathrm{He}$ at low and medium temperatures. Experimental data: $\star$ Johnston and Grilly [56]; • Becker et al. [57]; ○ Becker and Misenta [58]; $\triangle$ Coremans et al. [59]; ש Flynn et al. [48]; $\square$ Gracki et al. [49]; $\diamond$ Kao and Kobayashi [60]; $\mathbf{\nabla}$ Clarke and Smith [61]; $\nabla$ Gough et al. [62]; $\square$ Evers et al. [51]. Calculated values: - - Hurly and Mehl [2]. 
1

2 3 4 5 6

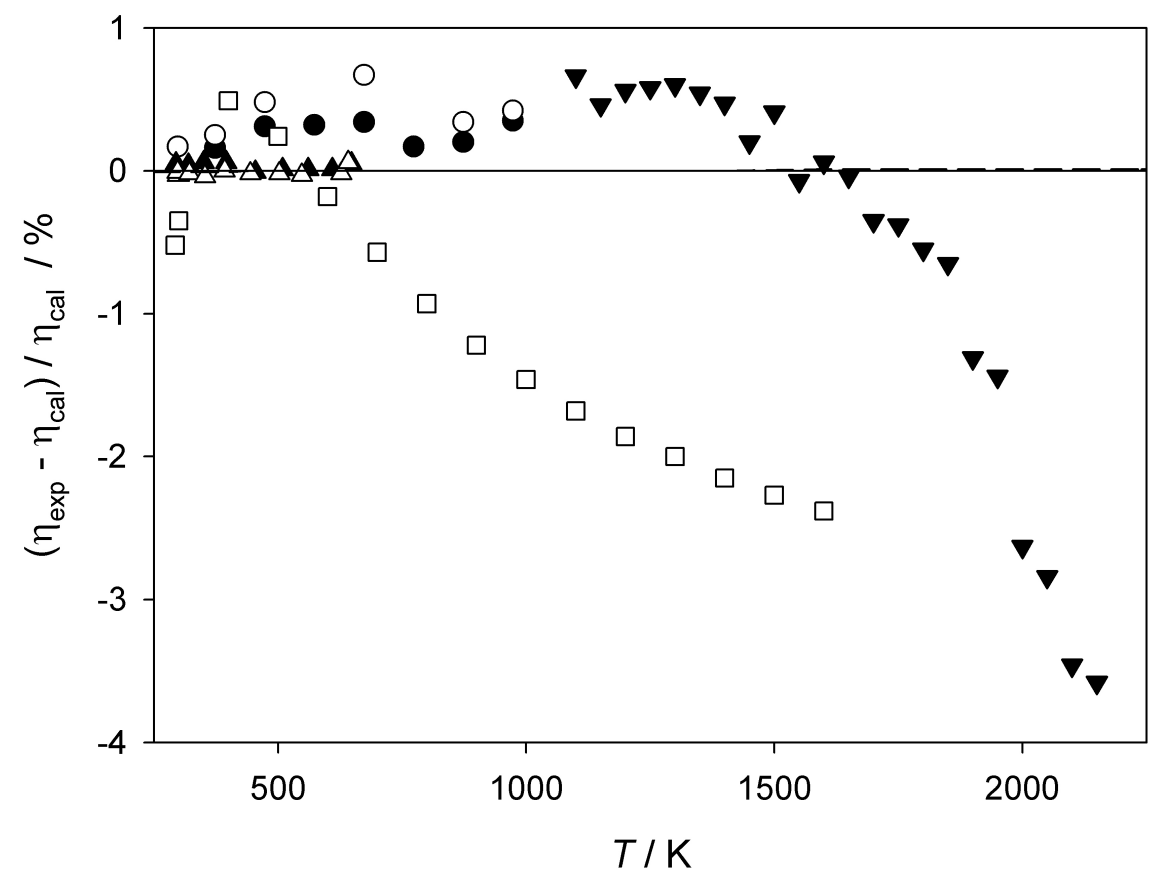

Figure 7. Deviations of experimental and calculated viscosity coefficients from values calculated with the new interatomic potential for ${ }^{4} \mathrm{He}$ at higher temperatures. Experimental data: $\bullet$ Kestin et al. [47]; ○ Kestin et al. [65]; $\boldsymbol{\nabla}$ Guevara et al. [66]; $\square$ Dawe and Smith [67]; $\boldsymbol{\Delta}$ Vogel [50], 1st series of measurements recalibrated; $\Delta$ Vogel [50], 2nd series of measurements recalibrated. Calculated values: - - - Hurly and Mehl [2]. 


\section{Page 29 of 47 \\ Molecular Physics}

II. Thermophysical standard values for low-density helium

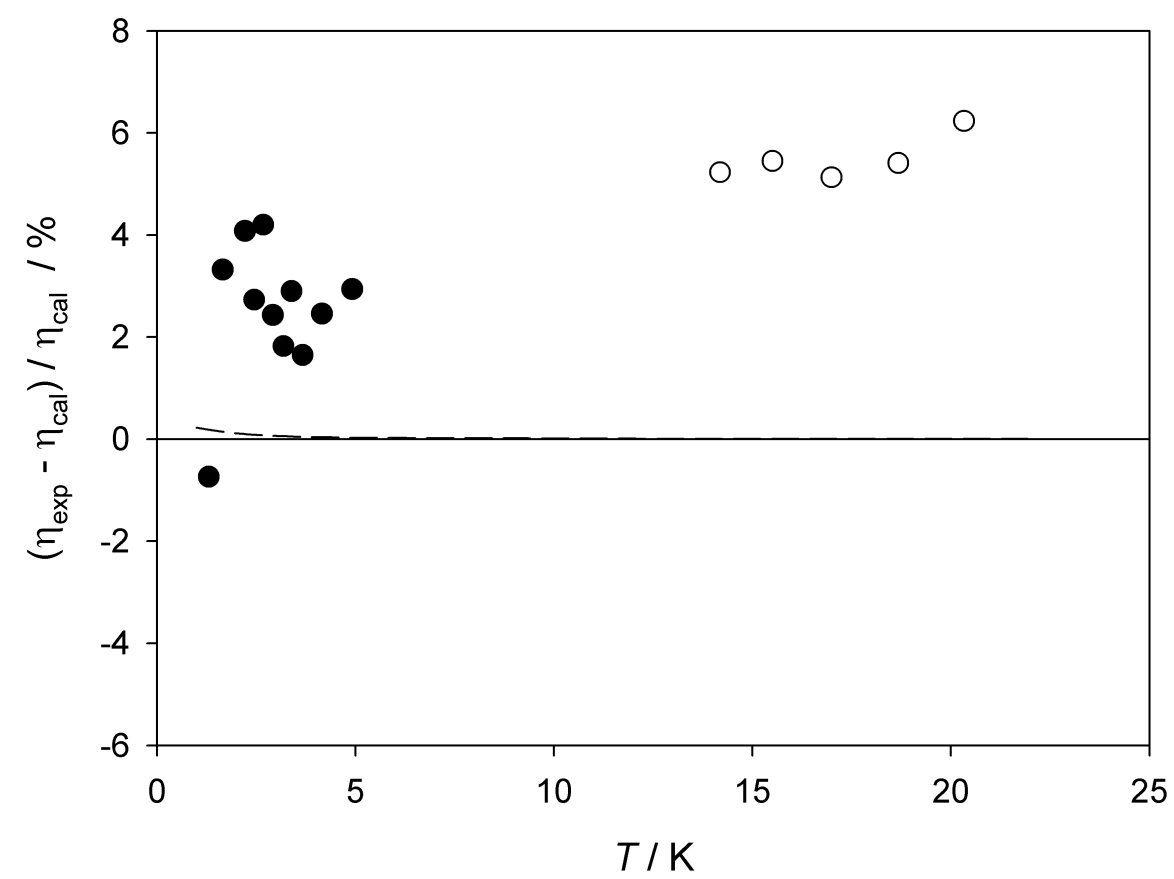

Figure 8. Deviations of experimental and calculated viscosity coefficients from values calculated with the new interatomic potential for ${ }^{3}$ He. Experimental data: • Becker et al. [57]; ○ Becker and Misenta [58]. Calculated values: - - Hurly and Mehl [2]. 
1

3

4

5

6

7

8

9

10

11

12

13

14

15

16

17

18

19

20

21

22

23

24

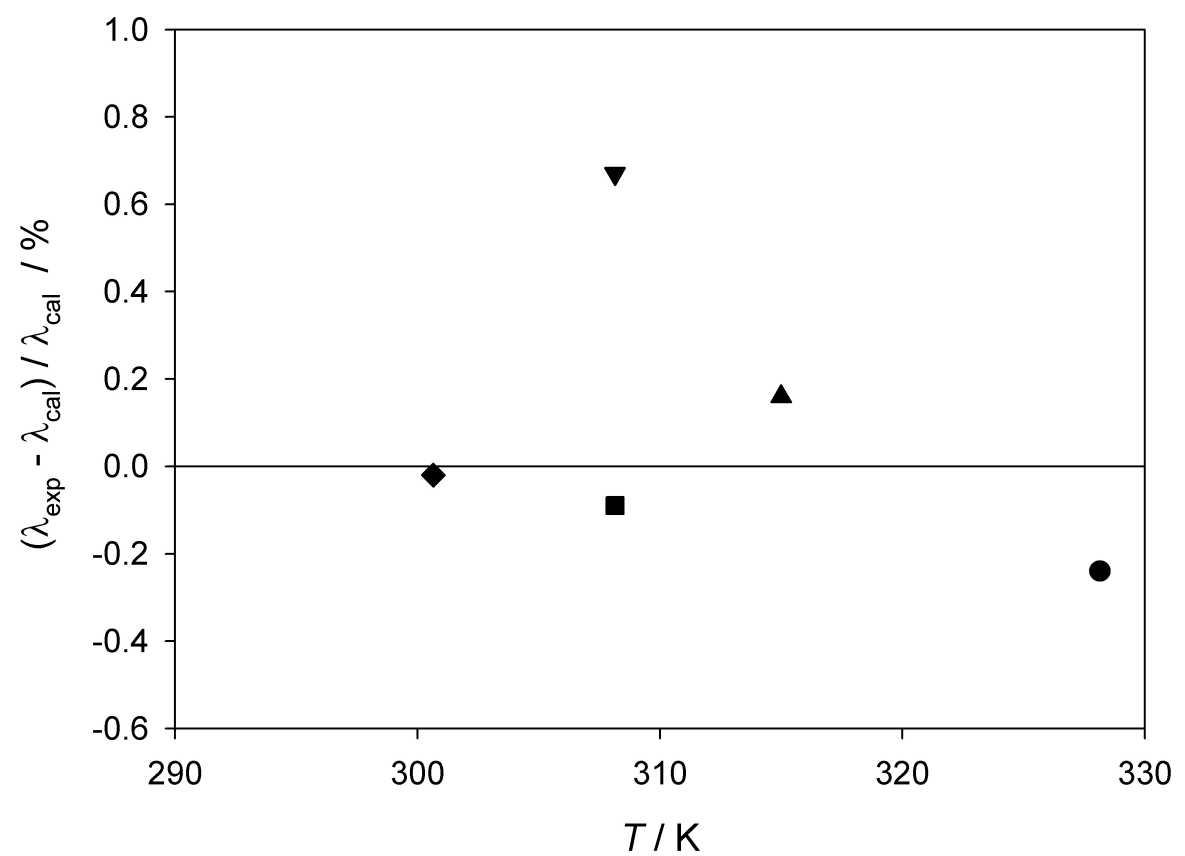

Figure 9. Deviations of experimental and calculated thermal conductivity coefficients from values calculated with the new interatomic potential for ${ }^{4} \mathrm{He}$ at room temperature. Experimental data: • Haarman [70]; Kestin et al. [71]; $\boldsymbol{\|}$ Assael et al. [72]; $\boldsymbol{\nabla}$ Mustafa et al. [73]; $\boldsymbol{\Delta}$ Johns et al. [74]. Calculated values: - - Hurly and Mehl [2]. 
1

2

3

4

5

6

7

8

9

10

11

12

13

14

15

16

17

18

19

20

21

22

23

24

25

26

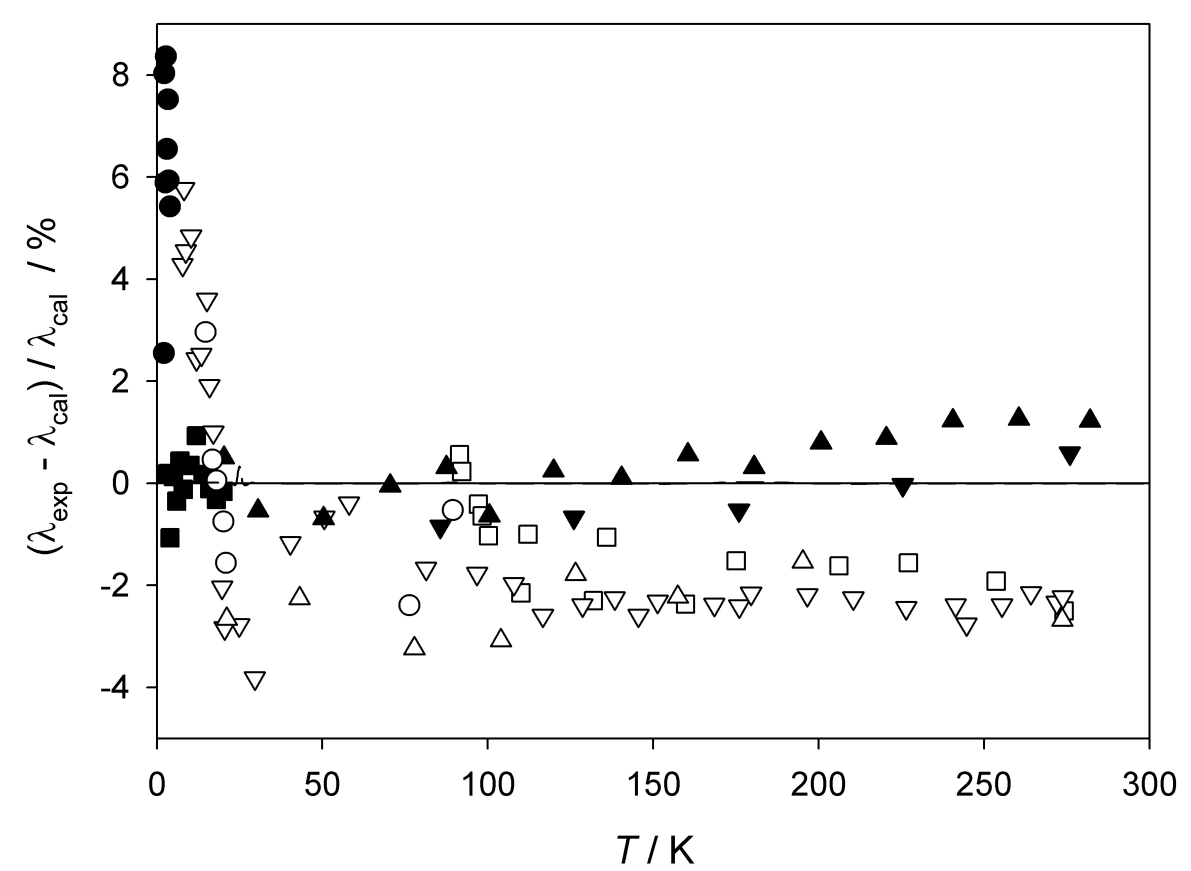

Figure 10. Deviations of experimental and calculated thermal conductivity coefficients from values calculated with the new interatomic potential for ${ }^{4} \mathrm{He}$ at low and medium temperatures. Experimental data: $\bigcirc$ Ubbink and de Haas [75]; $\triangle$ Golubev and Shpagina [76]; • Kerrisk and Keller [77]; $\boldsymbol{\Delta}$ Roder [78, 79]; $\square$ Shashkov et al. [80]; - Acton and Kellner [81]; $\nabla$ Popov and Zarev [82]; $\nabla$ Zarev et al. [83]. Calculated values: - - - Hurly and Mehl [2]. 


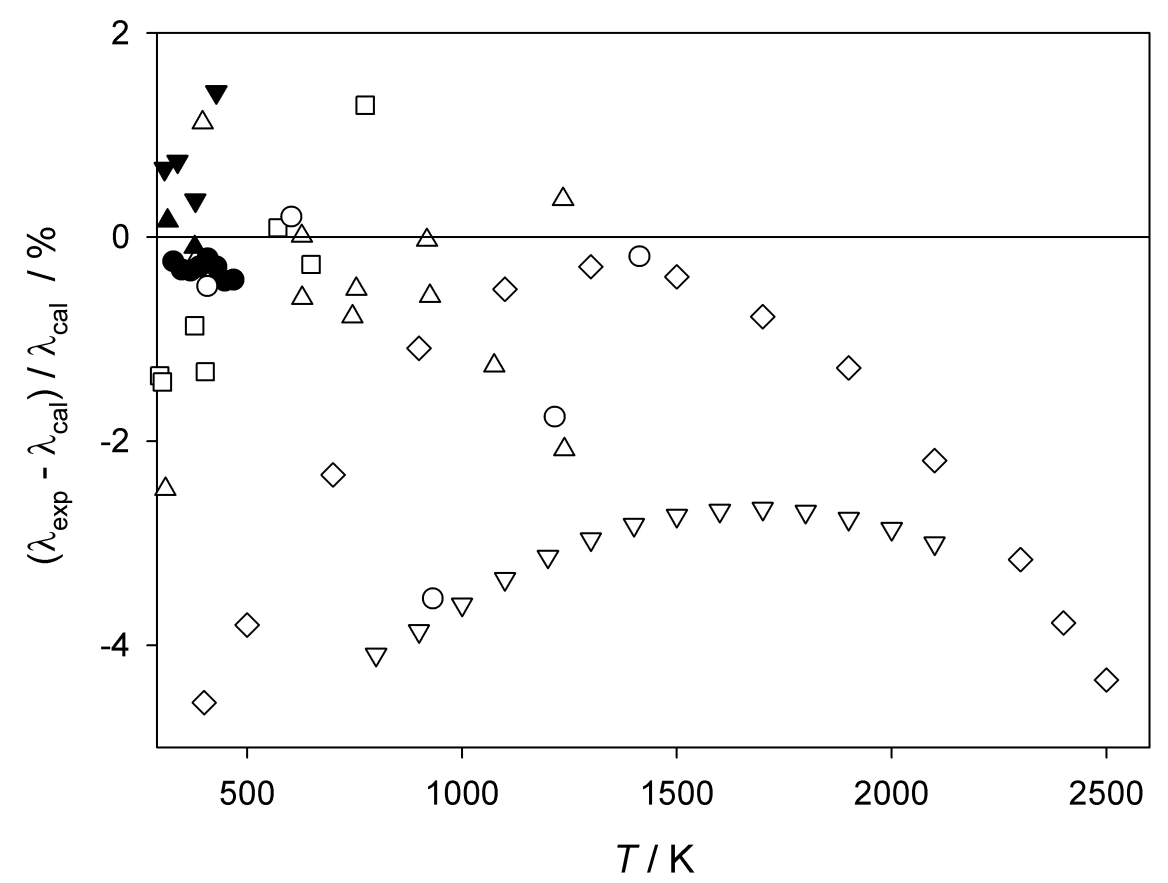

Figure 11. Deviations of experimental and calculated thermal conductivity coefficients from values calculated with the new interatomic potential for ${ }^{4} \mathrm{He}$ at higher temperatures. Experimental data: $\Delta$ Vargaftik and Zhimina [84]; $\square$ LeNeindre et al. [85]; • Haarman [70]; $\nabla$ Faubert and Springer [86]; $\bigcirc$ Martchenko and Shashkov [87]; $\diamond$ Jody et al. [88]; $\mathbf{\nabla}$ Mustafa et al. [73]; $\boldsymbol{\Delta}$ Johns et al. [74]. Calculated values: - - Hurly and Mehl [2]. 


\section{Page 33 of 47 \\ Molecular Physics}

II. Thermophysical standard values for low-density helium

3

4

5

6

9

10

11

12

13

14

15

16

17

18

19

20

21

22

23

24

25

26

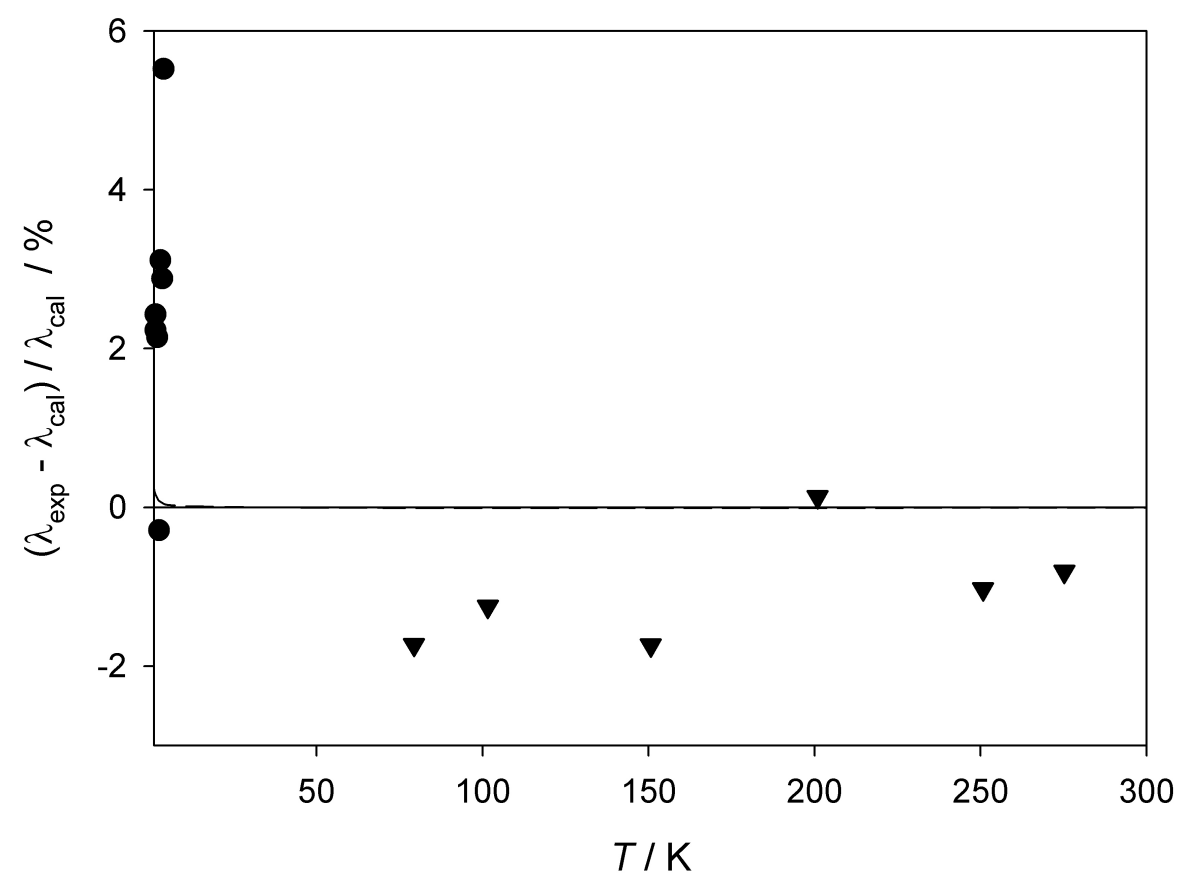

Figure 12. Deviations of experimental and calculated thermal conductivity coefficients from values calculated with the new interatomic potential for ${ }^{3}$ He. Experimental data: • Kerrisk and Keller [77]; v Zarev et al. [83]. Calculated values: - - Hurly and Mehl [2]. 
Appendix A: Thermophysical properties of ${ }^{4} \mathrm{He}$ and ${ }^{3} \mathrm{He}$ calculated in this work

Table A1. Thermophysical properties of ${ }^{4} \mathrm{He}$ and ${ }^{3} \mathrm{He}$ calculated in this work

\begin{tabular}{|c|c|c|c|c|c|c|c|}
\hline \multirow[b]{2}{*}{$T(\mathrm{~K})$} & \multicolumn{4}{|c|}{${ }^{4} \mathrm{He}$} & \multicolumn{3}{|c|}{${ }^{3} \mathrm{He}$} \\
\hline & $B\left(\mathrm{~cm}^{3} \mathrm{~mol}^{-1}\right)$ & $C\left(\mathrm{~cm}^{6} \mathrm{~mol}^{-2}\right)$ & $\eta(\mu \mathrm{Pas})$ & $\lambda\left(\mathrm{mW} \mathrm{m}^{-1} \mathrm{~K}^{-1}\right)$ & $B\left(\mathrm{~cm}^{3} \mathrm{~mol}^{-1}\right)$ & $\eta(\mu \mathrm{Pas})$ & $\lambda\left(\mathrm{mW} \mathrm{m}^{-1} \mathrm{~K}^{-1}\right)$ \\
\hline 1.00 & -475.93 & & 0.32875 & 2.6288 & -236.32 & 0.55936 & 5.7842 \\
\hline 1.20 & -370.40 & & 0.34015 & 2.7166 & -205.50 & 0.66407 & 6.8674 \\
\hline 1.40 & -302.50 & & 0.35796 & 2.8422 & -180.96 & 0.76250 & 7.8906 \\
\hline 1.60 & -255.41 & & 0.38408 & 3.0296 & -161.04 & 0.85015 & 8.8094 \\
\hline 1.80 & -220.88 & & 0.41793 & 3.2800 & -144.60 & 0.92505 & 9.6041 \\
\hline 2.00 & -194.45 & & 0.45824 & 3.5852 & -130.84 & 0.98722 & 10.274 \\
\hline 2.25 & -168.96 & & 0.51567 & 4.0268 & -116.54 & 1.0491 & 10.952 \\
\hline 2.50 & -149.15 & & 0.57869 & 4.5156 & -104.70 & 1.0969 & 11.485 \\
\hline 2.75 & -133.28 & & 0.64523 & 5.0334 & -94.764 & 1.1345 & 11.908 \\
\hline 3.00 & -120.24 & & 0.71357 & 5.5656 & -86.312 & 1.1651 & 12.254 \\
\hline 3.50 & -100.05 & & 0.85058 & 6.6326 & -72.723 & 1.2153 & 12.807 \\
\hline 4.00 & -85.089 & & 0.98279 & 7.6619 & -62.293 & 1.2603 & 13.281 \\
\hline 4.50 & -73.531 & & 1.1072 & 8.6316 & -54.042 & 1.3060 & 13.745 \\
\hline 5.00 & -64.323 & & 1.2234 & 9.5375 & -47.354 & 1.3546 & 14.231 \\
\hline 6.00 & -50.558 & & 1.4333 & 11.179 & -37.169 & 1.4609 & 15.290 \\
\hline 7.00 & -40.750 & & 1.6203 & 12.645 & -29.776 & 1.5756 & 16.444 \\
\hline 8.00 & -33.404 & & 1.7913 & 13.987 & -24.162 & 1.6940 & 17.647 \\
\hline 9.00 & -27.697 & & 1.9509 & 15.239 & -19.751 & 1.8127 & 18.864 \\
\hline 10.00 & -23.135 & & 2.1018 & 16.423 & -16.193 & 1.9303 & 20.074 \\
\hline 11.00 & -19.407 & & 2.2458 & 17.552 & -13.262 & 2.0456 & 21.265 \\
\hline 12.00 & -16.304 & & 2.3841 & 18.637 & -10.807 & 2.1583 & 22.431 \\
\hline 14.00 & -11.439 & & 2.6468 & 20.695 & -6.9255 & 2.3757 & 24.685 \\
\hline 16.00 & -7.8037 & & 2.8943 & 22.635 & -3.9990 & 2.5830 & 26.837 \\
\hline 18.00 & -4.9899 & & 3.1296 & 24.478 & -1.7171 & 2.7814 & 28.897 \\
\hline 20.00 & -2.7515 & 310.0 & 3.3548 & 26.242 & 0.10887 & 2.9722 & 30.878 \\
\hline 22.00 & -0.93187 & 291.5 & 3.5713 & 27.937 & 1.6004 & 3.1561 & 32.788 \\
\hline 23.00 & -0.14494 & 284.4 & 3.6767 & 28.763 & 2.2474 & 3.2458 & 33.720 \\
\hline 24.00 & 0.57370 & 278.0 & 3.7803 & 29.574 & 2.8394 & 3.3341 & 34.637 \\
\hline 25.00 & 1.2323 & 272.5 & 3.8823 & 30.373 & 3.3827 & 3.4212 & 35.541 \\
\hline 26.00 & 1.8377 & 267.4 & 3.9828 & 31.160 & 3.8829 & 3.5069 & 36.432 \\
\hline 28.00 & 2.9119 & 258.7 & 4.1795 & 32.700 & 4.7723 & 3.6750 & 38.178 \\
\hline 30.00 & 3.8346 & 251.2 & 4.3710 & 34.199 & 5.5378 & 3.8389 & 39.880 \\
\hline 35.00 & 5.6493 & 236.4 & 4.8302 & 37.794 & 7.0478 & 4.2327 & 43.969 \\
\hline 40.00 & 6.9740 & 225.1 & 5.2662 & 41.206 & 8.1528 & 4.6073 & 47.858 \\
\hline 45.00 & 7.9739 & 216.1 & 5.6832 & 44.469 & 8.9878 & 4.9661 & 51.583 \\
\hline 50.00 & 8.7482 & 208.6 & 6.0842 & 47.607 & 9.6342 & 5.3116 & 55.169 \\
\hline 60.00 & 9.8508 & 196.6 & 6.8472 & 53.575 & 10.552 & 5.9697 & 62.000 \\
\hline 70.00 & 10.578 & 187.1 & 7.5682 & 59.215 & 11.154 & 6.5924 & 68.462 \\
\hline 80.00 & 11.075 & 179.3 & 8.2558 & 64.592 & 11.561 & 7.1868 & 74.630 \\
\hline 90.00 & 11.425 & 172.6 & 8.9160 & 69.754 & 11.842 & 7.7579 & 80.555 \\
\hline 100.00 & 11.673 & 166.7 & 9.5531 & 74.735 & 12.038 & 8.3092 & 86.275 \\
\hline
\end{tabular}




\section{Page 35 of 47 \\ Molecular Physics}

1 Table A2. Table continued

2

$3 \quad T(\mathrm{~K}) \quad B\left(\mathrm{~cm}^{3} \mathrm{~mol}^{-1}\right) \quad C\left(\mathrm{~cm}^{6} \mathrm{~mol}^{-2}\right) \quad \eta(\mu \mathrm{Pas}) \quad \lambda\left(\mathrm{mW} \mathrm{m}^{-1} \mathrm{~K}^{-1}\right) \quad B\left(\mathrm{~cm}^{3} \mathrm{~mol}^{-1}\right) \quad \eta(\mu \mathrm{Pas}) \quad \lambda\left(\mathrm{mW} \mathrm{m}^{-1} \mathrm{~K}^{-1}\right)$

4

5

6
7
8

$120.00 \quad 11.977 \quad 156.8$

12.126

12.186

160.00

12.191

12.163

12.099

12.015

11.927

11.920

11.826

11.818

11.714

11.609

11.504

11.400

11.199

11.006

10.651

10.332

10.045

10.045
9.7857

9.5497

9.1348

8.7799

8.4711

8.1987

7.9556

7.4446

7.0330

6.6905

6.3988

6.1457

5.9229

5.5459

5.2363

4.9752

4.7505

4.5542

\begin{tabular}{cc}
156.8 & 10.770 \\
148.5 & 11.926 \\
141.5 & 13.032 \\
135.4 & 14.099 \\
129.9 & 15.130 \\
123.9 & 16.378 \\
118.7 & 17.588 \\
114.3 & 18.678 \\
114.0 & 18.764 \\
110.0 & 19.826 \\
109.8 & 19.910 \\
105.9 & 21.030 \\
102.4 & 22.128 \\
99.22 & 23.204 \\
96.27 & 24.261 \\
90.98 & 26.325 \\
86.38 & 28.331 \\
78.73 & 32.196 \\
72.56 & 35.905 \\
67.46 & 39.488 \\
63.15 & 42.966 \\
59.44 & 46.357 \\
53.36 & 52.922 \\
48.56 & 59.253 \\
44.65 & 65.398 \\
41.38 & 71.390 \\
38.60 & 77.253 \\
33.17 & 91.461 \\
29.16 & 105.17 \\
26.06 & 118.52 \\
23.58 & 131.56 \\
21.55 & 144.38 \\
19.84 & 157.00 \\
17.14 & 181.80 \\
15.08 & 206.12 \\
13.46 & 230.11 \\
12.14 & 253.83 \\
11.05 & 277.35 \\
& \\
\hline
\end{tabular}

84.250
93.283
101.93
110.26
118.32
128.07
137.52
146.04
146.71
155.01
155.66
164.41
172.98
181.39
189.64
205.76
221.42
251.60
280.55
308.51
335.66
362.12
413.35
462.75
510.68
557.42
603.15
713.95
820.87
924.88
1026.6
1126.5
1224.9
1418.1
1607.6
1794.5
1979.3
2162.5

12.267

12.364

9.3631

12.386

10.364

97.208

97.208
107.59

$12.364-11.248$

$\begin{array}{lll}12.314 & 13.142 & 136.39\end{array}$

$\begin{array}{lll}12.229 & 14.225 & 147.62\end{array}$

$\begin{array}{lll}12.128 & 15.274 & 158.51\end{array}$

$\begin{array}{lll}12.028 & 16.220 & 168.30\end{array}$

$\begin{array}{lll}12.020 & 16.294 & 169.07\end{array}$

$\begin{array}{lll}11.916 & 17.215 & 178.63\end{array}$

$\begin{array}{lll}11.908 & 17.288 & 179.38\end{array}$

$\begin{array}{lll}11.795 & 18.260 & 189.46\end{array}$

$\begin{array}{lll}11.682 & 19.212 & 199.32\end{array}$

$\begin{array}{lll}11.571 & 20.146 & 209.00\end{array}$

$\begin{array}{lll}11.462 & 21.064 & 218.51\end{array}$

$\begin{array}{lll}11.462 & 21.064 & 218.51 \\ 11.252 & 22.855 & 237.07\end{array}$

$\begin{array}{lll}11.053 & 24.596 & 255.11\end{array}$

$\begin{array}{lll}10.688 & 27.951 & 289.87\end{array}$

$\begin{array}{lll}10.362 & 31.170 & 323.22\end{array}$

$\begin{array}{lll}10.071 & 34.279 & 355.43\end{array}$

$\begin{array}{lll}9.8077 & 37.299 & 386.70\end{array}$

$\begin{array}{lll}9.5689 & 40.242 & 417.18\end{array}$

$\begin{array}{lll}9.1500 & 45.940 & 476.20\end{array}$

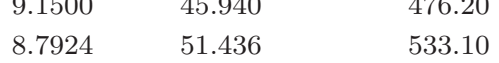

$\begin{array}{lll}8.4816 & 56.771 & 588.32\end{array}$

$\begin{array}{lll}8.2078 & 61.972 & 642.17\end{array}$

$\begin{array}{lll}7.9636 & 67.062 & 694.85\end{array}$

$\begin{array}{lll}7.4506 & 79.395 & 822.50\end{array}$

$\begin{array}{lll}7.4506 & 79.395 & 822.50 \\ 7.0379 & 91.299 & 945.67\end{array}$

$\begin{array}{lll}6.6945 & 102.88 & 1065.5\end{array}$

$\begin{array}{lll}6.4022 & 114.21 & 1182.7\end{array}$

$\begin{array}{lll}6.1486 & 125.33 & 1297.7\end{array}$

$\begin{array}{lll}6.1486 & 125.33 & 1411.1\end{array}$

1633.6

$\begin{array}{lll}5.5480 & 157.81 & 1633.6 \\ 5.2379 & 178.92 & 1852.0\end{array}$

$\begin{array}{lll}4.9765 & 199.75 & 2067.2\end{array}$

$\begin{array}{lll}4.7519 & 220.34 & 2280.1\end{array}$

$\begin{array}{lll}4.5551 & 240.76 & 2491.2\end{array}$ 


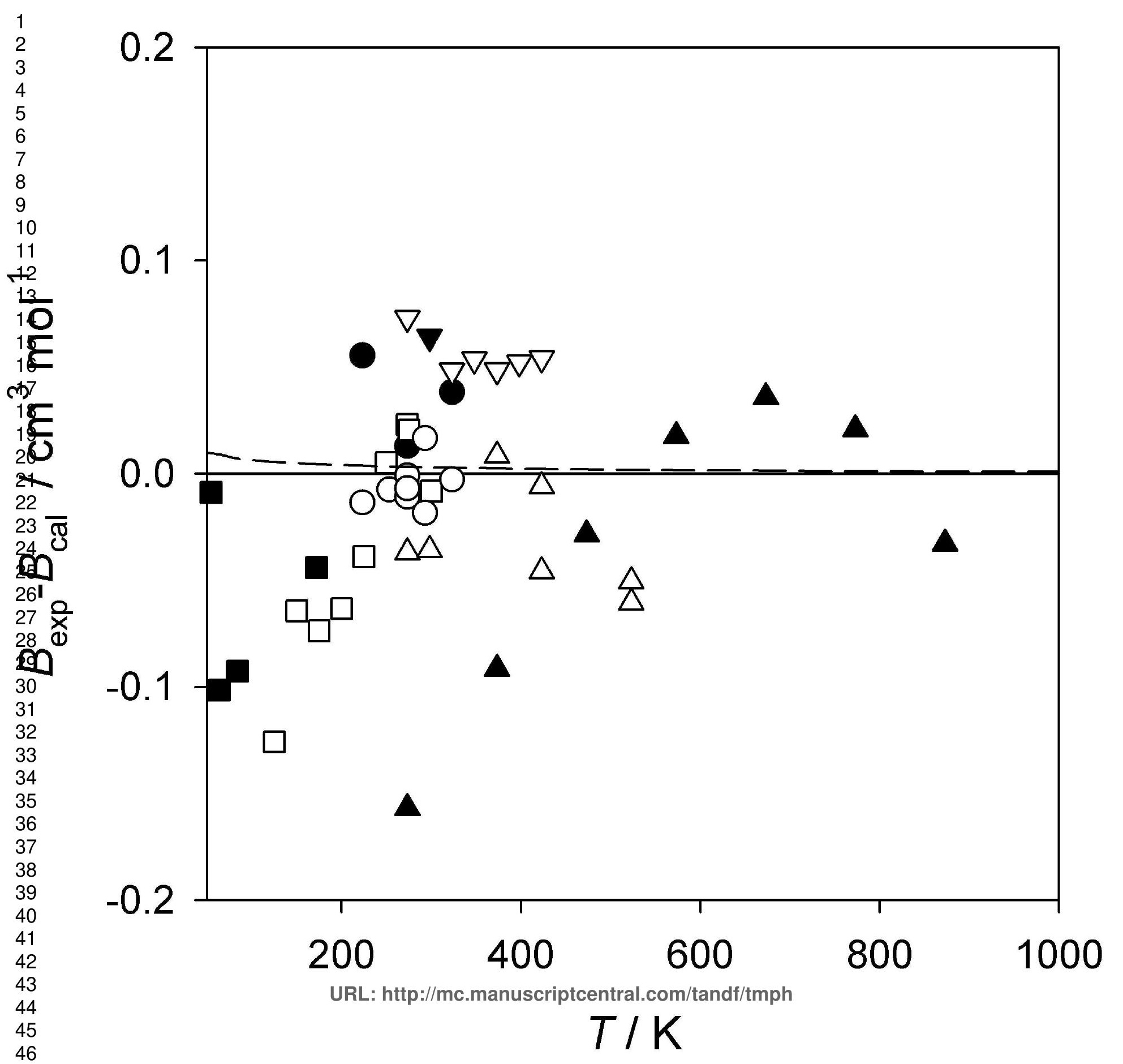




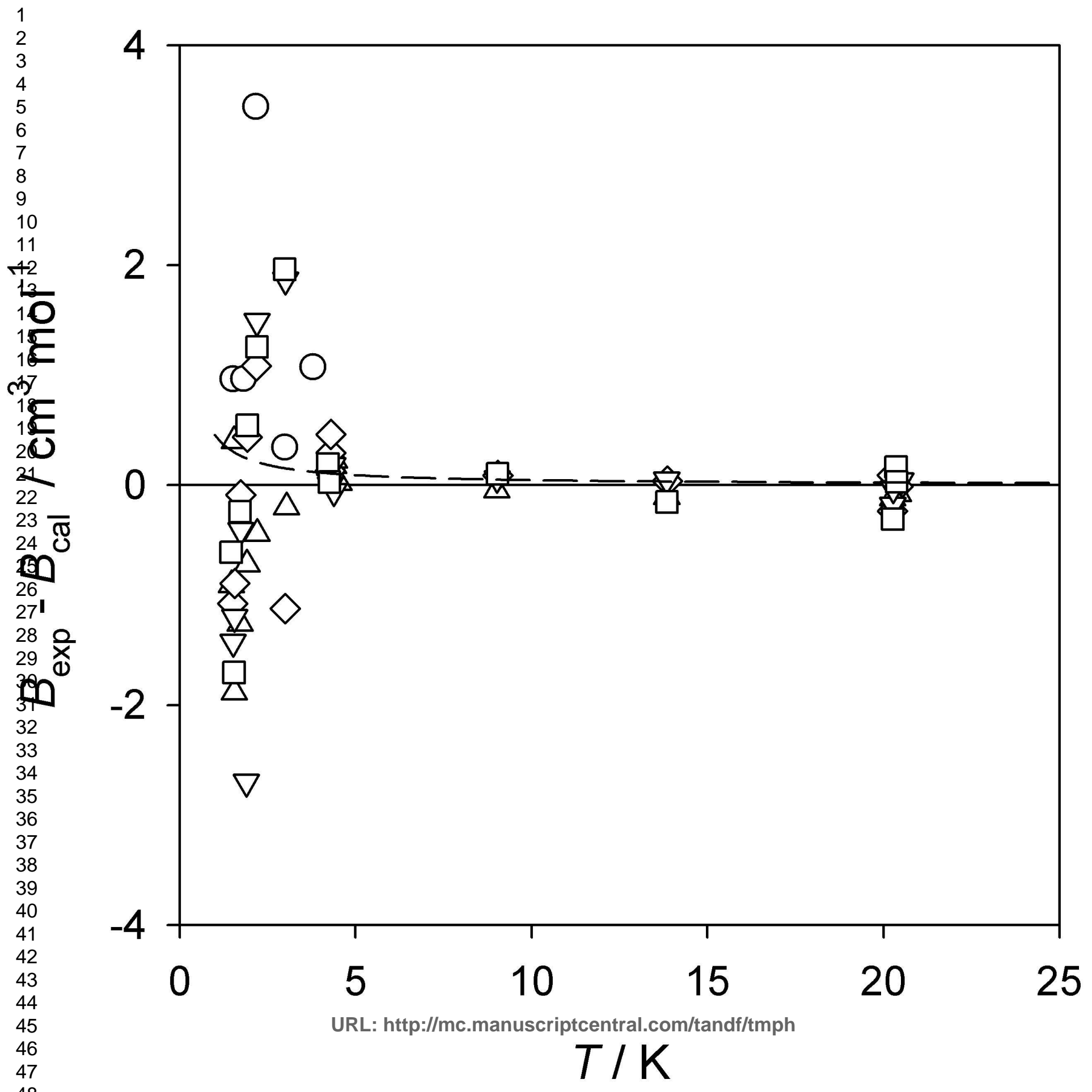




\section{Page 39 of 47}

Molecular Physics

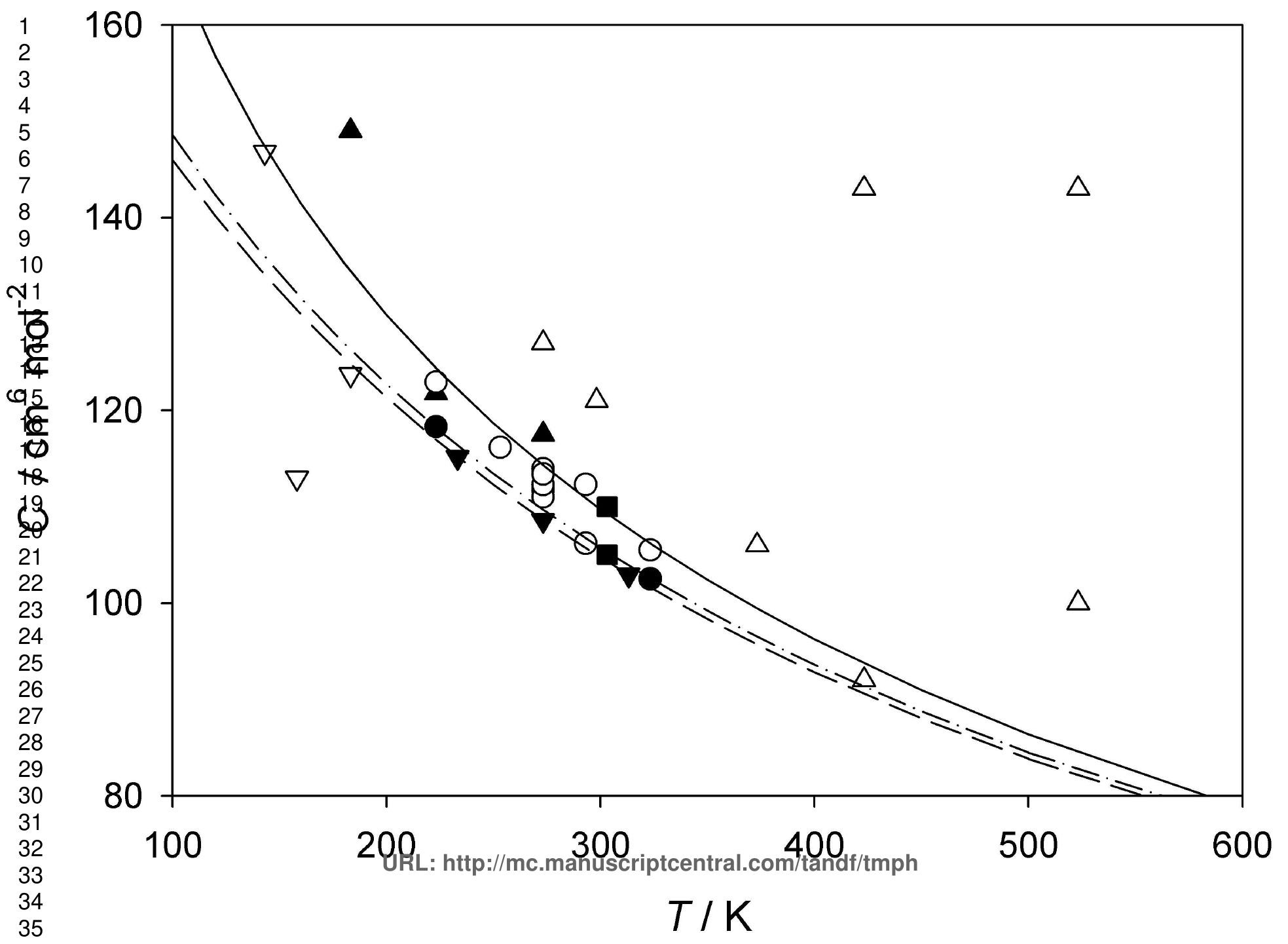




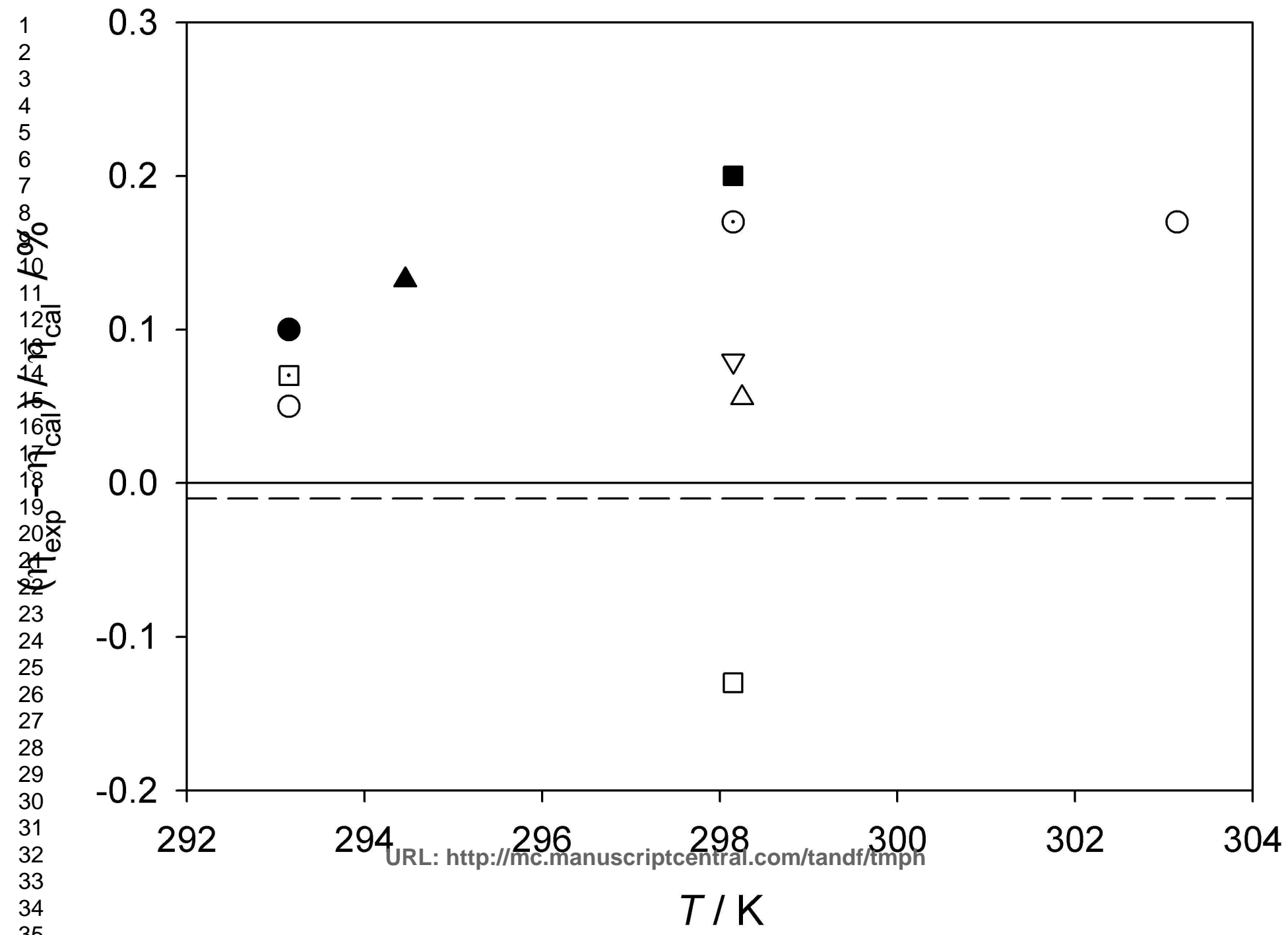




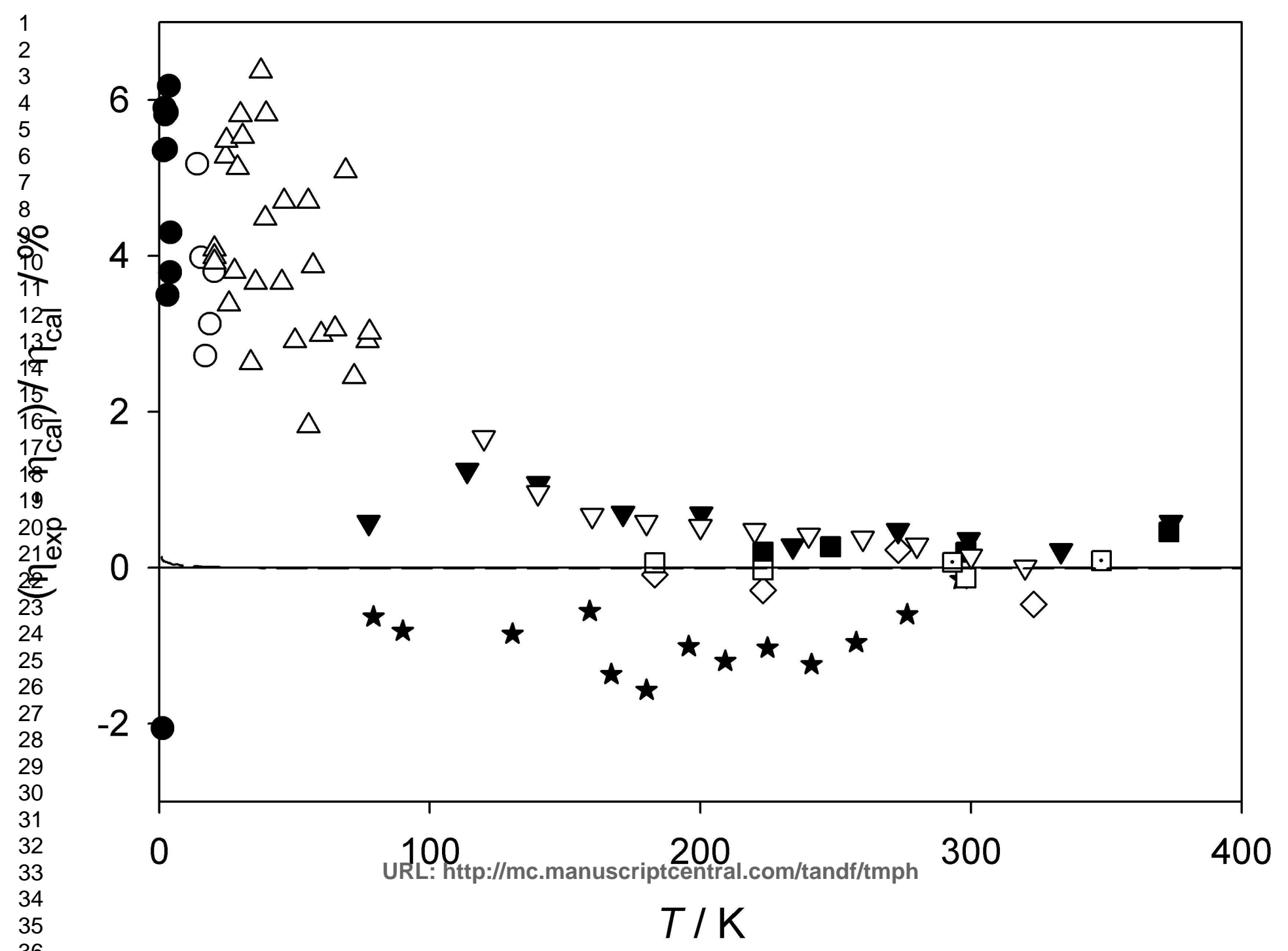




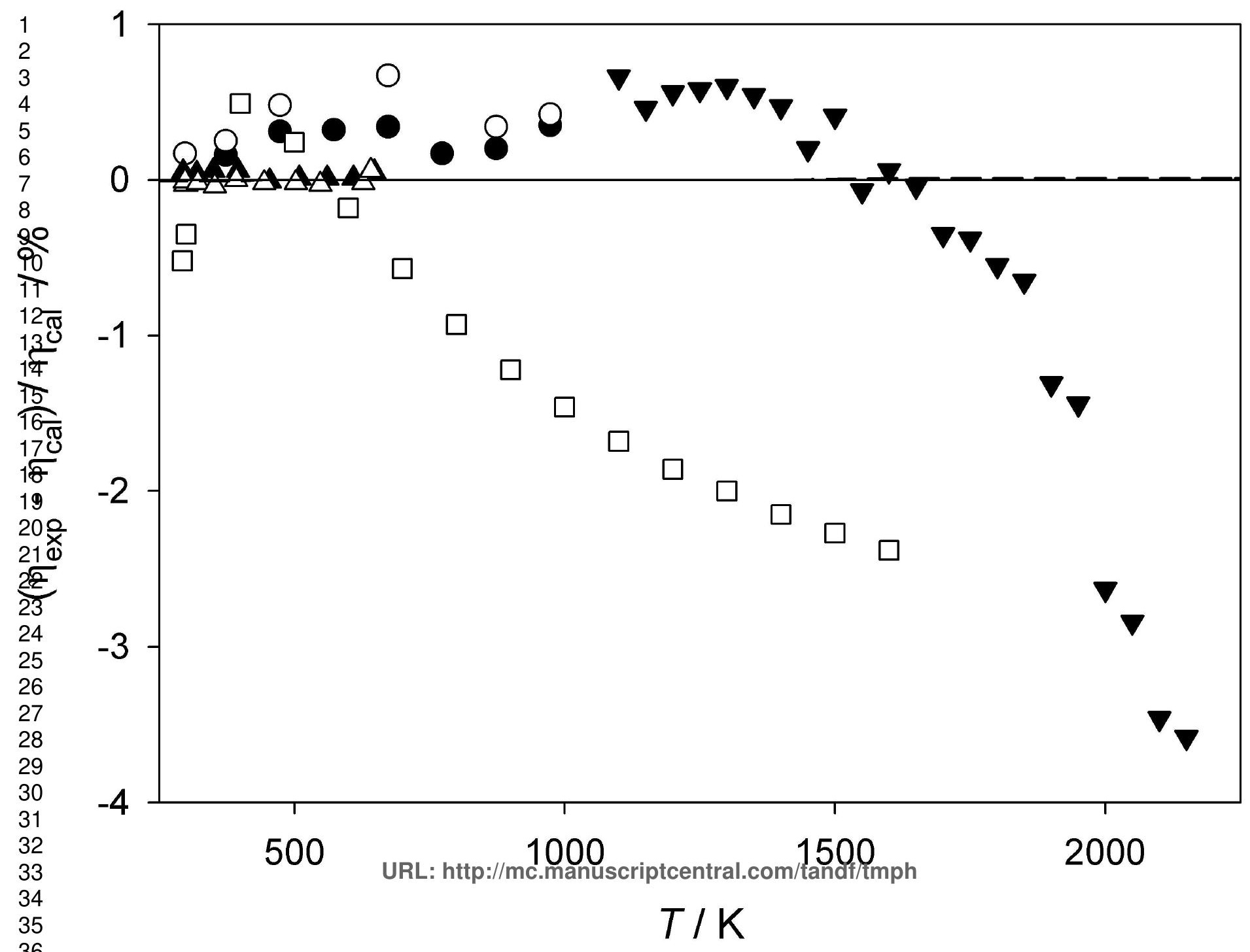




\section{Page 43 of 47}

Molecular Physics

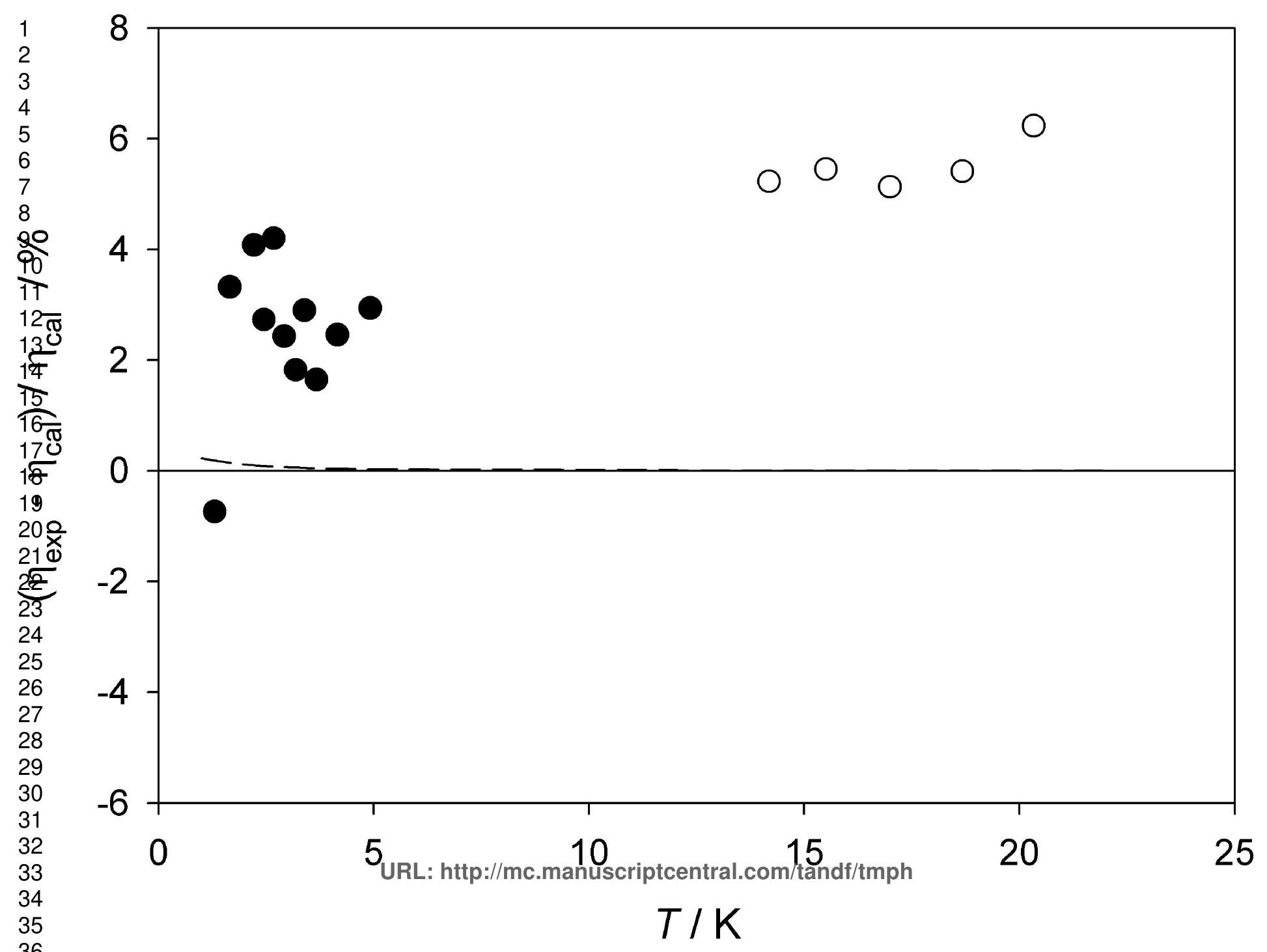




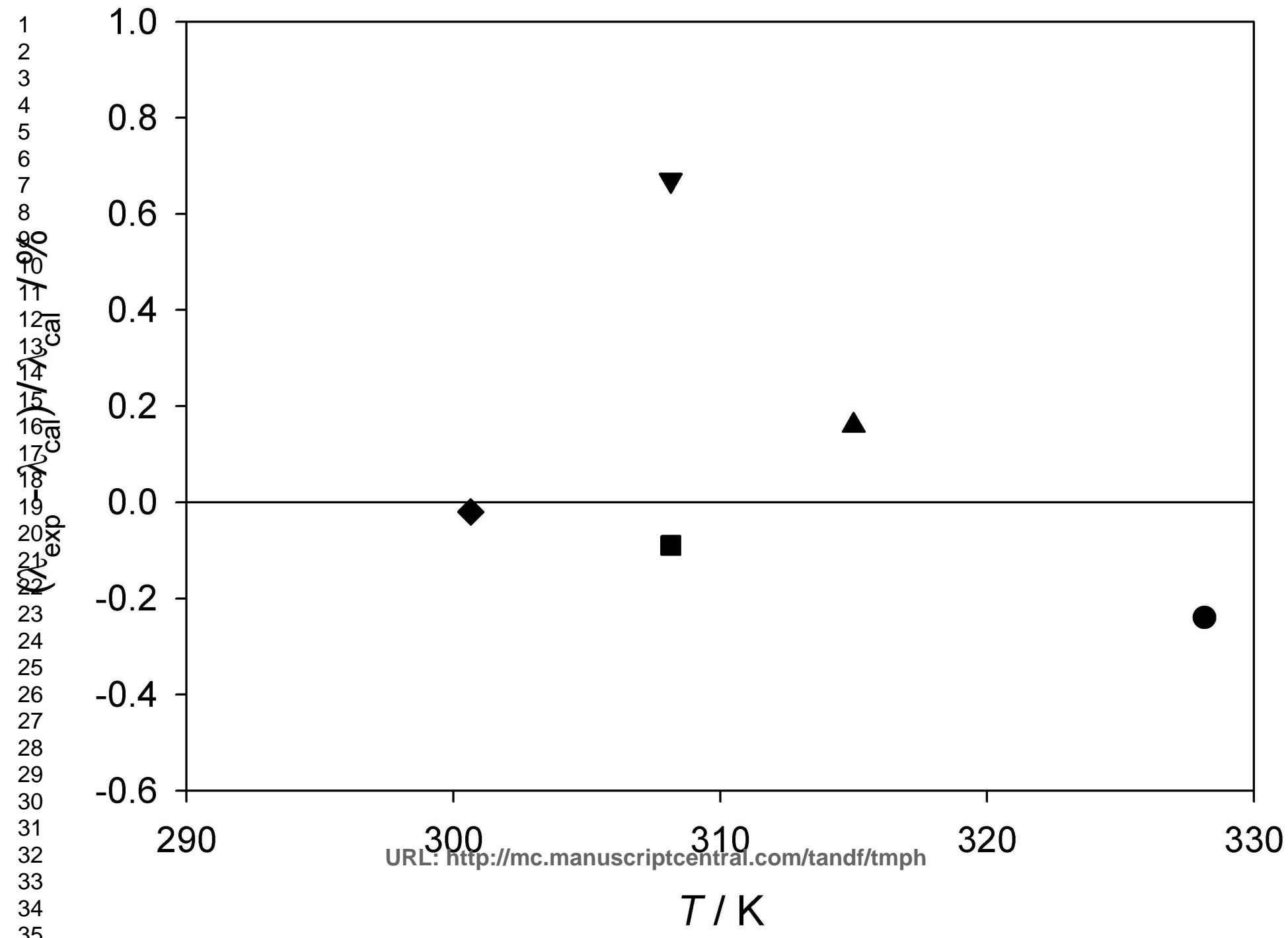




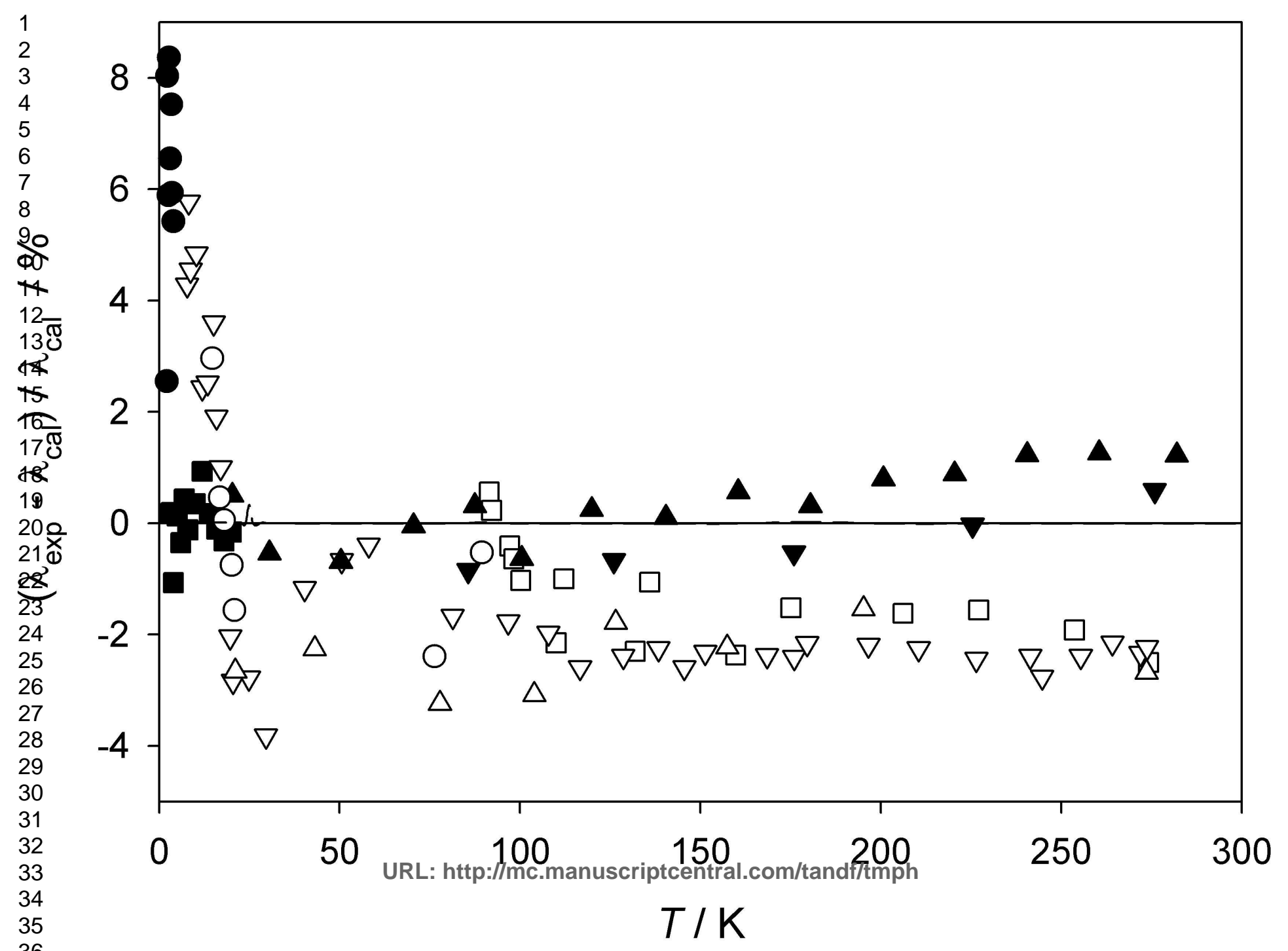


